\title{
Vibration of a beam on continuous elastic foundation with nonhomogeneous stiffness and damping under a harmonically excited \\ mass
}

\author{
S.G. Koroma*, M.F.M. Hussein**, J.S. Owen \\ Centre for Structural Engineering and Construction (CSEC), Faculty of Engineering, University of Nottingham, \\ University Park, Nottingham, NG7 2RD, UK
}

\begin{abstract}
In this paper, a proposed method of analysis of a beam that is continuously supported on a linear nonhomogeneous elastic foundation and subjected to a harmonically excited mass is presented. The solution is obtained by decomposing the nonhomogeneous foundation properties and the beam displacement response into double Fourier summations which are solved in the frequency-wavenumber domain, from which the space-time domain response can be obtained. The method is applied to railway tracks with step variation in foundation properties. The validity of this method is checked, through examples, against existing methods for both homogeneous and nonhomogeneous foundation parameters. The effect of inhomogeneity and the magnitude of the mass are also investigated. It is found that a step variation in foundation properties leads to a reduction in the beam displacement and an increase in the resonance frequency, for increasing step change, with the reverse occurring for decreasing s. Furthermore, a beam on nonhomogeneous foundation may exhibit multiple resonances corresponding to the foundation stiffness of individual sections, as the mass moves through the respective sections along the beam.
\end{abstract}

Keywords: Nonhomogeneous foundation, Double Fourier Summation, continuously supported beam, harmonically excited mass, frequency-wavenumber domain

\section{Introduction}

The model of a beam on an elastic foundation has been extensively used over the years for modelling the dynamic behaviour of railway tracks [1, 2, 3]. Diverse solutions have been presented for this problem for varying types of loads; static and dynamic, stationary and moving, deterministic and stochastic. In their solutions, most authors assume the beam is supported on a linear elastic foundation with homogeneous stiffness and damping parameters. Solutions to the homogeneous case are readily available in the literature, for e.g. the Fourier and Laplace transformation methods as well as direct approaches of solving the differential equations. Although this fundamental assumption of homogeneous parameters provides good understanding of the dynamic behaviour of a beam on an elastic foundation, it limits the true representation of the practical situation in most railway based applications. This is because there are many practical instances of nonhomogeneity in railway tracks. For example, in Hunt [4] several classes of inhomogeneity/roughness in railway tracks including variations in track-bed profile, foundation stiffness, sleeper spacing are given.

\footnotetext{
${ }^{*}$ Corresponding author: Tel: +44 (0) 115951 3288, Email: evxsgk1@nottingham.ac.uk

** Now at ISVR, University of Southampton, Southampton, SO17 1BJ, UK

Email addresses: M.hussein@soton.ac.uk (M.F.M. Hussein), john.owen@nottingham.ac.uk (J.S. Owen) 
The effect of spatial variation of track stiffness on the vibration of infinite beams on elastic foundation has been studied by several authors, mostly with the use of perturbation techniques for relatively small variations. Mahmoud and Tawil [5] analysed the quasi-static response of beams on random elastic foundation using truncated power series expansion of the random displacements. Dynamic effects were later accounted for by Frýba et al. [6] who used a firstorder perturbation technique with stochastic finite elements to obtain the steady state solution of an infinite beam on a random foundation with uncertain damping, subjected to a constant moving force. The results, which are given in the form of variances of the deflection and bending moment of the beam, show that the coefficient of variation of deflection is larger than that of the bending moment at the point of application of the force, with randomness of the foundation stiffness being of greater significance than the uncertainty in the damping. Andersen and Nielsen [7] analysed an infinite beam resting on a Kelvin foundation with the inclusion of a shear layer and subjected to a moving SDOF vehicle. The spatial variation of the foundation vertical stiffness is described by a stochastic homogeneous field consisting of small random variations around a predefined mean value. A first-order perturbation analysis was proposed to establish the relationship between the variation of the spring stiffness and the responses of the vehicle mass and the beam. The accuracy of this method, however, depends on the speed of the vehicle as well as the degree of variation in the random track stiffness, with fairly poor results obtained for speeds and stiffness variations over $30 \%$. Verichev and Metrikine [8] studied the instability of a mass moving along a beam that is supported on an inhomogeneous elastic foundation with periodically varying stiffness. Perturbation analysis was used to obtain analytic expressions for the vibration conditions of the beam to become unstable. In all these cases, only small variations in track stiffness and damping have been considered by the authors in order to guarantee the accuracy of their solutions when making use of the perturbation technique. For example, in [6], the coefficients of variation in stiffness and damping are kept small enough compared to unity; i.e. $|\epsilon| \ll 1$ and $|\gamma| \ll 1$ respectively. The same assumption is also emphasized in [8] where the small parameter $\mu \ll 1$, for the same reason stated above. Also, most of these models only considered variation in stiffness whereas the damping in treated as constant. However, large levels of inhomogeneity can be present in railway track supporting structures; for e.g. moving from one track type to another as from a ballasted track to a bridge structure or vice versa.

Other methods have also been employed in studying beams on nonhomogeneous foundations. Pavlović and Wylie [9] investigated the free vibration of a beam on Winkler foundation with linearly varying modulus along the beam span using a power series approach. They concluded that the free response is mainly divided into two regions; up to a certain value of the stiffness below which the response of the beam can be determined by averaging the linearly varying stiffness and adopting equivalent homogeneous models and the region beyond this value in which this cannot be done. Wave propagation in a beam on a Winkler foundation with random spatial variation of spring stiffness has been studied by Schevenels et al [10] with the focus on understanding the influence of correlation length. Their results show that even small spatial variations can have an influence on the response at large distance from the source if the correlation length and the wavelength are of the same order of magnitude.

Generally, there are two categories of methods used in solving the differential equation governing the dynamic behaviour of a beam on elastic foundation. The first category is based on discretisation techniques such as the finite element and finite difference methods carried out in the space-time domain, whereas the second group adopts transformation techniques in the frequency and/or wavenumber domains; see for e.g. Knothe and Grassie [11]. Advances in discretisation methods have been immense with applications to solving beams on linear homogeneous foundations; see for e.g. [12], nonhomogeneous foundations; see for e.g. [13], [14, nonlinear foundations; see for e.g. [15], etc. However, the applications of frequencywavenumber domain methods have been limited to linear homogeneous problems or periodically 
nonhomogeneous ones, see for e.g. [16. It is intended in this work to extend the applications of frequency-wavenumber domain technique to incorporate other forms of nonhomogeneity in the differential equation.

In this paper, an alternative approach is proposed for analysing a beam on elastic foundation with nonhomogeneous stiffness and damping under a moving harmonically excited mass. The method addresses some of the limitations of the aforementioned methods as large levels of variation in both foundation stiffness and damping are considered. The effects of nonhomogeneity on the beam response are analysed. In section 2 , the model is presented together with the generalised differential equation describing the beam dynamic behaviour, and the proposed method of solution, involving the use of Fourier sums is presented in section 3. The method is applied to railway tracks with continuous elastic foundation with step variation in properties in section 4. Results are then presented in section 5, including validation of the current method against existing methods such as the Fourier transformation method for homogeneous parameters and also standard finite elements approach in the space-time domain.

\section{Model formulation}

Figure 1(a) shows an infinitely long Euler-Bernoulli beam supported on a continuous linear elastic foundation resting on a rigid base. The vertical foundation stiffness and damping are modelled, using springs and dashpots respectively, as generally nonhomogeneous in the spatial domain along the length of the beam. The beam is traversed by a vehicle, excited by an oscillating load, at constant velocity in the direction shown. The vehicle is represented only by
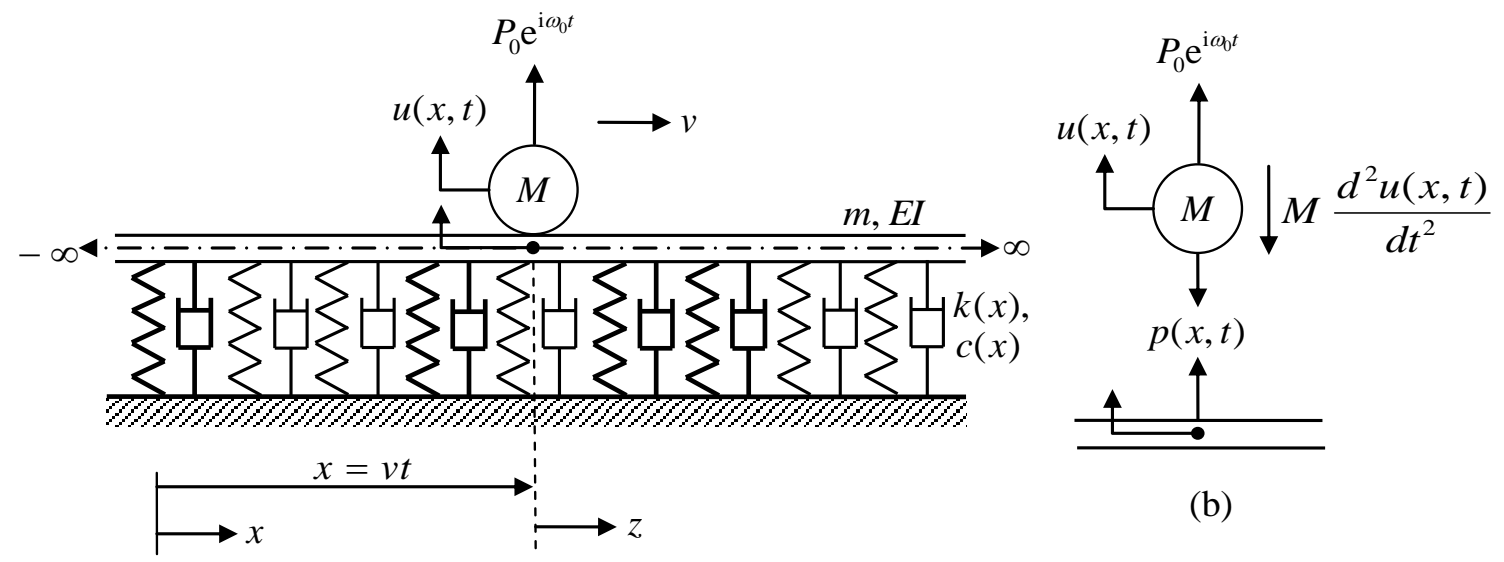

(b)

(a)

Figure 1: (a) Moving mass on an Euler-Bernoulli beam on nonhomogeneous elastic foundation, (b) equilibrium conditions at the mass-beam interface

a mass, assumed to be a simplification of a train in which only the unsprung part is included, i.e. assuming the suspensions isolate the dynamics of the sprung components of the vehicle in the frequency range of interest. The general boundary conditions associated with the infinite beam theory are adopted here; i.e. the deflection, slope and bending moment at an infinitely long distance, on either side of the moving load, vanish at all times.

\subsection{Equation of motion}

Fig. 1(b) shows the equilibrium conditions at the mass-beam interface. It is assumed that perfect contact between the mass and the beam is maintained at all times. The partial differential equation of motion governing the dynamic behaviour of the beam on nonhomogeneous 
elastic foundation subjected to an arbitrary force, $p(x, t)$, can be written as

$$
E I \frac{\partial^{4} u(x, t)}{\partial x^{4}}+m \frac{\partial^{2} u(x, t)}{\partial t^{2}}+c(x) \frac{\partial u(x, t)}{\partial t}+k(x) u(x, t)=p(x, t),
$$

where $E I$ and $m$ are the bending stiffness and mass per unit length of the rail respectively, $k(x)$ and $c(x)$ are the nonhomogeneous foundation stiffness and damping respectively; $u(x, t)$ is the displacement of the rail at any point, $x$, at an instant, $t$. The external force, $p(x, t)$, in this case is the mutual force at the mass-beam interface and can be obtained from the equilibrium of the mass such that

$$
p(x, t)=\left[P_{0} \mathrm{e}^{\mathrm{i} \omega_{0} t}-M \frac{d^{2} u(x, t)}{d t^{2}}\right] \delta(x-v t),
$$

where $M$ is the magnitude of the moving mass; $P_{0}$ and $\omega_{0}$ are the amplitude and angular frequency of the harmonic load, moving with speed, $v ; \omega_{0}=2 \pi f_{0}, f_{0}$ being the excitation frequency and $\delta($.$) is the Dirac delta function. For convenience, the harmonic load has been$ expressed in a complex form (i.e. $\mathrm{e}^{\mathrm{i} \omega_{0} t}$ ), i being the imaginary unit with value of $\sqrt{-1}$.

Substituting Eq. (2) into Eq. (1) and replacing the full derivative associated with the moving frame with its equivalent partial derivatives in the fixed frame, results in

$$
\begin{aligned}
& E I \frac{\partial^{4} u(x, t)}{\partial x^{4}}+m \frac{\partial^{2} u(x, t)}{\partial t^{2}}+c(x) \frac{\partial u(x, t)}{\partial t}+k(x) u(x, t) \\
& =\left[P_{0} \mathrm{e}^{\mathrm{i} \omega_{0} t}-M\left(\frac{\partial^{2} u(x, t)}{\partial t^{2}}+2 v \frac{\partial^{2} u(x, t)}{\partial x \partial t}+v^{2} \frac{\partial^{2} u(x, t)}{\partial x^{2}}\right)\right] \delta(x-v t) .
\end{aligned}
$$

Equation (3) is best solved, using the proposed method, when the delta function on its Right Hand Side (RHS) is expressed only as a function of time. This is achieved by transforming it from the fixed frame of reference $(x, t)$ to the moving frame of reference $(z=x-v t, t)$, see [1. Consider the following transformation of the partial derivatives between the two frames of reference, see for e.g. [7]

$$
\begin{aligned}
& \partial / \partial x=\partial / \partial z ; \quad \partial / \partial t=\partial /\left.\partial t\right|_{z}-v \partial / \partial z ; \\
& \partial^{2} / \partial t^{2}=\partial^{2} /\left.\partial t^{2}\right|_{z}-2 v \partial^{2} / \partial z \partial t+v^{2} \partial^{2} / \partial z^{2} ;
\end{aligned}
$$

Applying Eq. (4) to Eq. (3), Eq. (3) can be rewritten in the moving frame of reference as follows

$$
\begin{aligned}
& E I \frac{\partial^{4} u(z, t)}{\partial z^{4}}+m\left(\frac{\partial^{2} u(z, t)}{\partial t^{2}}-2 v \frac{\partial^{2} u(z, t)}{\partial z \partial t}+v^{2} \frac{\partial^{2} u(z, t)}{\partial z^{2}}\right) \\
& +c(z, t)\left(\frac{\partial u(z, t)}{\partial t}-v \frac{\partial u(z, t)}{\partial z}\right)+k(z, t) u(z, t)=\left[P_{0} \mathrm{e}^{\mathrm{i} \omega_{0} t}-M \frac{\partial^{2} u(z, t)}{\partial t^{2}}\right] \delta(z)
\end{aligned}
$$

Equation (5) is the coupled differential equation for a moving mass on a beam on nonhomogeneous elastic foundation. The solution procedure, using the proposed method, is presented in the next section.

\section{Solution of the equation of motion}

The procedure for solving Eq. (5) involves decomposing the nonhomogeneous foundation stiffness and damping; $k(z, t)$ and $c(z, t)$, the displacement response of the beam $u(z, t)$ and the external forcing function into DFS forms. 


\subsection{Formulation of the Double Fourier Sum (DFS)}

Let the beam displacement $u(z, t)$ be continuous on $z$, on the interval $z \in\left\{-z_{m}, z_{m}\right\}$, deferring for a moment the dependence of $u$ on time $t$, with $z_{m}$ being the maximum distance on either side of the moving load. It should be noted that $z_{m}$ must be chosen large enough for a given set of excitation frequency and load speed in order to satisfy the prevailing boundary conditions stated in section (2). For the purpose of performing Fourier transformation on $u(z, t)$ from the space domain to the wavenumber domain, it is assumed that $u(z, t)$ is zero outside the predefined boundaries of $z$, hence the Fourier transform needs only be performed on the specified limits of $z$. With this supposition, the Fourier transform of $u(z, t)$ in the wavenumbertime domain $\hat{u}(\xi, t)$ is

$$
\hat{u}(\xi, t)=\int_{-z_{m}}^{z_{m}} u(z, t) \mathrm{e}^{-\mathrm{i} \xi z} \mathrm{~d} z,
$$

where $\xi$ is the wavenumber, $\xi=2 \pi / \lambda ; \lambda$ being the wavelength of each harmonic component of the response.

Now considering the dependence of $u$ on time $t$, the temporal Fourier transformation on $\hat{u}(\xi, t)$ to the frequency domain results in the wavenumber-frequency domain counterpart of $u(z, t)$ as follows

$$
\tilde{u}(\xi, \omega)=\int_{-t_{m}}^{t_{m}} \int_{-z_{m}}^{z_{m}} u(z, t) \mathrm{e}^{-\mathrm{i} \xi z} \mathrm{e}^{-\mathrm{i} \omega t} \mathrm{~d} z \mathrm{~d} t,
$$

where $\omega$ is the angular frequency of each harmonic component of the response and $t_{m}$ is the maximum time included in the computation.

The corresponding inverse double Fourier transform of Eq. (7) is given as

$$
u(z, t)=\frac{1}{4 \pi^{2}} \int_{-\omega_{m}}^{\omega_{m}} \int_{-\xi_{m}}^{\xi_{m}} \tilde{u}(\xi, \omega) \mathrm{e}^{\mathrm{i} \xi z} \mathrm{e}^{\mathrm{i} \omega t} \mathrm{~d} \xi \mathrm{d} \omega,
$$

where $\xi_{m}$ and $\omega_{m}$ are respectively the maximum wavenumber and frequency included in the computation.

Similar expressions can be written for the nonhomogeneous stiffness and damping as functions of their wavenumber-frequency domain counterparts as in Eq. (9) and (10) below:

$$
\begin{aligned}
& k(z, t)=\frac{1}{4 \pi^{2}} \int_{-\omega_{m}}^{\omega_{m}} \int_{-\xi_{m}}^{\xi_{m}} \tilde{k}(\xi, \omega) \mathrm{e}^{\mathrm{i} \xi z} \mathrm{e}^{\mathrm{i} \omega t} \mathrm{~d} \xi \mathrm{d} \omega, \\
& c(z, t)=\frac{1}{4 \pi^{2}} \int_{-\omega_{m}}^{\omega_{m}} \int_{-\xi_{m}}^{\xi_{m}} \tilde{c}(\xi, \omega) \mathrm{e}^{\mathrm{i} \xi z} \mathrm{e}^{\mathrm{i} \omega t} \mathrm{~d} \xi \mathrm{d} \omega .
\end{aligned}
$$

The Fourier transform of $\delta(z)$ on the RHS of Eq. (5) results in unity, which means that the inverse transform can be expressed simply as a function of wavenumber as

$$
\delta(z)=\frac{1}{2 \pi} \int_{-\xi_{m}}^{\xi_{m}} \mathrm{e}^{\mathrm{i} \xi z} \mathrm{~d} \xi .
$$

Since the system is linear, we can back substitute Eqs. (8)-(11) into Eq. (5); the resulting equation is an integral equation that governs the beam dynamics, with unknowns being the 
response amplitudes in the $\xi-\omega$ domain as follows

$$
\begin{aligned}
& \frac{1}{4 \pi^{2}} \int_{-\omega_{m}}^{\omega_{m}} \int_{-\xi_{m}}^{\xi_{m}}\left(E I \xi^{4}-m(\omega-\xi v)^{2}\right) \tilde{u}(\xi, \omega) \mathrm{e}^{\mathrm{i} \xi z} \mathrm{e}^{\mathrm{i} \omega t} \mathrm{~d} \xi \mathrm{d} \omega \\
& -\left(\frac{1}{4 \pi^{2}} \int_{-\omega_{m}}^{\omega_{m}} \int_{-\xi_{m}}^{\xi_{m}} M \omega^{2} \tilde{u}(\xi, \omega) \mathrm{e}^{\mathrm{i} \xi z} \mathrm{e}^{\mathrm{i} \omega t} \mathrm{~d} \xi \mathrm{d} \omega\right)\left(\frac{1}{2 \pi} \int_{-\xi_{m}}^{\xi_{m}} \mathrm{e}^{\mathrm{i} \xi z} \mathrm{~d} \xi\right) \\
& +\left(\frac{1}{4 \pi^{2}} \int_{-\omega_{m}}^{\omega_{m}} \int_{-\xi_{m}}^{\xi_{m}}(\mathrm{i}(\omega-\xi v) \tilde{c}(\xi, \omega)+\tilde{k}(\xi, \omega)) \mathrm{e}^{\mathrm{i} \xi z} \mathrm{e}^{\mathrm{i} \omega t} \mathrm{~d} \xi \mathrm{d} \omega\right) \\
& \times\left(\frac{1}{4 \pi^{2}} \int_{-\omega_{m}}^{\omega_{m}} \int_{-\xi_{m}}^{\xi_{m}} \tilde{u}(\xi, \omega) \mathrm{e}^{\mathrm{i} \xi z} \mathrm{e}^{\mathrm{i} \omega t} \mathrm{~d} \xi \mathrm{d} \omega\right)=\frac{P_{0} \mathrm{e}^{\mathrm{i} \omega_{0} t}}{2 \pi} \int_{-\xi_{m}}^{\xi_{m}} \mathrm{e}^{\mathrm{i} \xi z} \mathrm{~d} \xi
\end{aligned}
$$

In Eq. (12), the presence of the nonhomogeneous foundation properties couples the response at different frequencies and wavenumbers thus preventing a simplified solution for $\tilde{u}(\xi, \omega)$. Therefore the inverse Fourier transforms in Eq. 12 must be evaluated, and this is most simply done using numerical integration such as the trapezium rule.

Dividing the wavenumber-frequency domain into an evenly spaced grid with $2 \alpha+1$ points on the interval $\{-\alpha \Delta \xi, \alpha \Delta \xi\}$ and $2 \beta+1$ points on the interval $\{-\beta \Delta \omega, \beta \Delta \omega\}$, where $\alpha$ and $\beta$ are integers, yields the wavenumber and frequency sequences given below

$$
\begin{aligned}
\xi_{p} & =p \Delta \xi ; \text { for } p \in\{-\alpha, \alpha\} ; \text { where } \Delta \xi=\pi / z_{m}, \\
\omega_{q} & =q \Delta \omega ; \text { for } q \in\{-\beta, \beta\} ; \text { where } \Delta \omega=\pi / t_{m} .
\end{aligned}
$$

Using Eq. (13a) and (13b), the integrals in Eq. (12) can be replaced by approximate Fourier sums to obtain the algebraic form in Eq. (14), which governs the beam behaviour with the unknowns being the response amplitudes in with the $\xi-\omega$ domain

$$
\begin{aligned}
& \sum_{q=-\beta}^{\beta} \sum_{p=-\alpha}^{\alpha}\left(E I \xi_{p}^{4}-m\left(\omega_{q}-\xi_{p} v\right)^{2}\right) U_{p, q} \mathrm{e}^{\mathrm{i} \xi_{p} z} \mathrm{e}^{\mathrm{i} \omega_{q} t} \\
& +\sum_{e=-\tau}^{\tau} \sum_{d=-\sigma}^{\sigma} \sum_{s=-\beta}^{\beta} \sum_{r=-\alpha}^{\alpha}\left(\mathrm{i}\left(\omega_{s}-\xi_{r} v\right) C_{d, e}+K_{d, e}\right) U_{r, s} \mathrm{e}^{\mathrm{i} \xi_{d+r} z} \mathrm{e}^{\mathrm{i} \omega_{e+s} t} \\
& -D \sum_{o=-\gamma}^{\gamma} \sum_{b=-\beta}^{\beta} \sum_{r=-\alpha}^{\alpha} M \omega_{b}^{2} U_{r, b} \mathrm{e}^{\mathrm{i} \xi_{o+r} z} \mathrm{e}^{\mathrm{i} \omega_{b} t}=P_{0} \sum_{q=-\beta}^{\beta} \sum_{p=-\alpha}^{\alpha} G_{p, q} \mathrm{e}^{\mathrm{i} \xi_{p} z} \mathrm{e}^{\mathrm{i} \omega_{q} t} .
\end{aligned}
$$

Note that $r$ and $b, s$ are synonymous indices to $p$ and $q$ respectively, but have been differentiated from the latter in order to conveniently account for the contributions of the nonhomogeneous components and the presence of the mass to the response amplitudes $U_{p, q} ; D$ is an integration constant $(=\Delta \xi / 2 \pi)$ and the index $o$ is associated with the transformation of the delta function on the LHS of Eq. (12), differentiated from that on the RHS for convenience.

In defining the parameters in Eq. (14), it is essential to show how they relate to their respective continuous integral forms given in Eqs. (8) to (11).

The double inverse Fourier transform of the response given in Eq. (8) can be recast in DFS form as follows

$$
u(z, t) \approx \sum_{q=-\beta}^{\beta} \sum_{p=-\alpha}^{\alpha} U_{p, q} \mathrm{e}^{\mathrm{i} \xi_{p} z} \mathrm{e}^{\mathrm{i} \omega_{q} t}, U_{p, q}=\frac{\tilde{\mathbf{u}}\left(\xi_{p}, \omega_{q}\right) \Delta \xi \Delta \omega}{4 \pi^{2}} .
$$


Similarly, DFS expressions can be written for the nonhomogeneous stiffness and damping given in Eqs. (9) and (10) such that

$$
k(z, t) \approx \sum_{e=-\tau}^{\tau} \sum_{d=-\sigma}^{\sigma} K_{d, e} \mathrm{e}^{\mathrm{i} \xi_{d} z} \mathrm{e}^{\mathrm{i} \omega_{e} t}, K_{d, e}=\frac{\tilde{\mathbf{k}}\left(\xi_{d}, \omega_{e}\right) \Delta \xi \Delta \omega}{4 \pi^{2}}
$$

and

$$
c(z, t) \approx \sum_{e=-\tau}^{\tau} \sum_{d=-\sigma}^{\sigma} C_{d, e} \mathrm{e}^{\mathrm{i} \xi_{d} z} \mathrm{e}^{\mathrm{i} \omega_{e} t}, C_{d, e}=\frac{\tilde{\mathbf{c}}\left(\xi_{d}, \omega_{e}\right) \Delta \xi \Delta \omega}{4 \pi^{2}}
$$

where $\tilde{\mathbf{u}}\left(\xi_{p}, \omega_{q}\right), \tilde{\mathbf{k}}\left(\xi_{d}, \omega_{e}\right)$ and $\tilde{\mathbf{c}}\left(\xi_{d}, \omega_{e}\right)$ are the respective double Fourier transformations of $u(z, t), k(z, t)$ and $c(z, t)$ from the $z-t$ domain to the $\xi-\omega$ domain.

In order to conform to the general DFS form, the forcing function on the RHS of Eq. (12) is also expressed as a Fourier sum as follows

$$
\frac{\mathrm{e}^{\mathrm{i} \omega_{0} t}}{2 \pi} \int_{-\xi_{m}}^{\xi_{m}} \mathrm{e}^{\mathrm{i} \xi z} \mathrm{~d} \xi \approx \sum_{q=-\beta}^{\beta} \sum_{p=-\alpha}^{\alpha} G_{p, q} \mathrm{e}^{\mathrm{i} \xi_{p} z} \mathrm{e}^{\mathrm{i} \omega_{q} t}
$$

where

$$
G_{p, q}= \begin{cases}0 & \text { for } \omega_{q} \neq \omega_{0} \\ \Delta \xi / 2 \pi & \text { for } \omega_{q}=\omega_{0}\end{cases}
$$

This implies that for all range of wavenumbers $\xi_{p}$, the external load is only concentrated at the excitation frequency.

Equation (14) can be simplified by adopting the following restrictive conditions

$$
d+r=p, e+s=q ; \quad-\alpha \leq r \leq \alpha,-\beta \leq s \leq \beta,
$$

and additionally specifying that $o+r=p$ and $b=q$, such that the summation indices $d, o$ and $e$ are simply replaced by $p-r$ and $q-s$ respectively as in Eq. 21.

$$
\begin{aligned}
& \sum_{q=-\beta}^{\beta} \sum_{p=-\alpha}^{\alpha}\left(E I \xi_{p}^{4}-m\left(\omega_{q}-\xi_{p} v\right)^{2}\right) U_{p, q} \mathrm{e}^{\mathrm{i} \xi_{p} z} \mathrm{e}^{\mathrm{i} \omega_{q} t} \\
& +\sum_{s=-\beta}^{\beta} \sum_{r=-\alpha}^{\alpha}\left(\mathrm{i}\left(\omega_{s}-\xi_{r} v\right) C_{p-r, q-s}+K_{p-r, q-s}\right) U_{r, s} \mathrm{e}^{\mathrm{i} \xi_{p} z} \mathrm{e}^{\mathrm{i} \omega_{q} t} \\
& -D \sum_{q=-\beta}^{\beta} \sum_{r=-\alpha}^{\alpha} M \omega_{q}^{2} U_{r, q} \mathrm{e}^{\mathrm{i} \xi_{p} z} \mathrm{e}^{\mathrm{i} \omega_{q} t}=P_{0} \sum_{q=-\beta}^{\beta} \sum_{p=-\alpha}^{\alpha} G_{p, q} \mathrm{e}^{\mathrm{i} \xi_{p} z} \mathrm{e}^{\mathrm{i} \omega_{q} t} .
\end{aligned}
$$

Equation (20) strictly restricts the formulation to within the boundaries of the $\xi-\omega$ domain mesh system and no contribution from values outside this region is considered. It is therefore important that the size of this mesh is large enough to encompass all significant values.

\subsection{Computation of $U_{p, q}$}

The calculation for $U_{p, q}$ is done numerically. A $2 \mathrm{D}$ mesh similar to the one shown in Fig. 2 is generated by specifying the range of frequencies and wavenumbers to be included in the computation, based on the range of $z$ and $t$, see Hussein [17] for example. The range of frequencies should be centred at the excitation frequency and the wavenumbers centred at zero, i.e. $\left\{\omega_{0}-\beta \Delta \omega: \Delta \omega: \omega_{0}+\beta \Delta \omega\right\}$ and $\{-\alpha \Delta \xi: \Delta \xi: \alpha \Delta \xi\}$ respectively. This is because, for a linear system, the largest response amplitudes are concentrated around the excitation frequency. In 


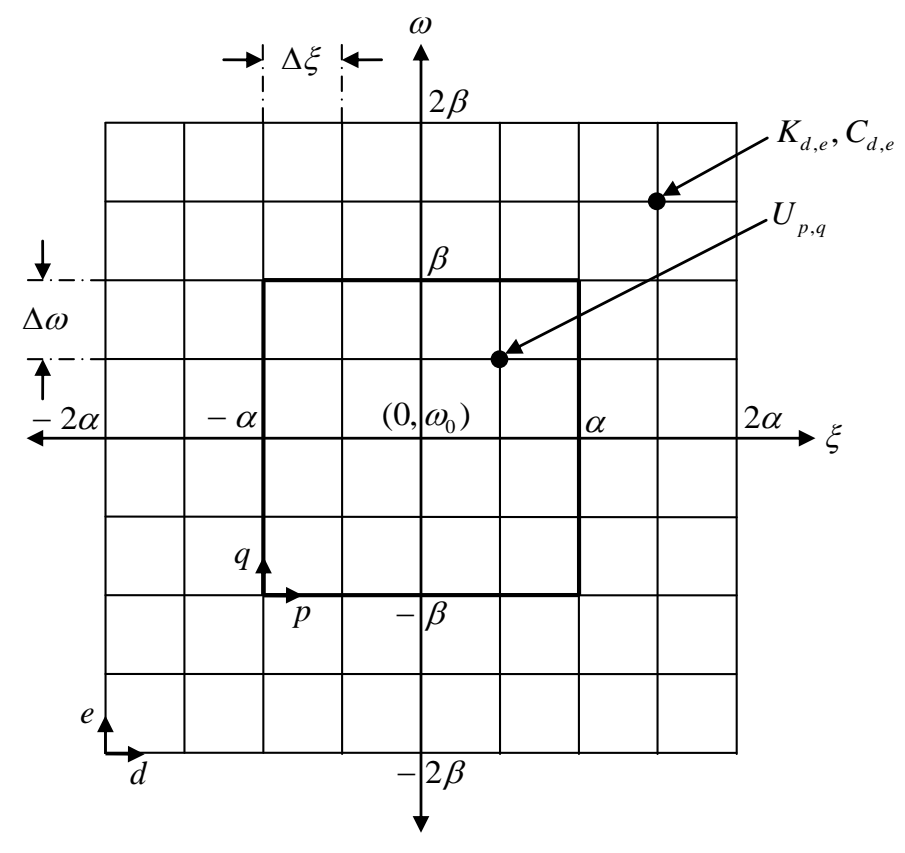

Figure 2: A 2D mesh for the computation of $U_{p, q}, K_{d, e}$ and $C_{d, e}$

order to obtain accurate results, these range of values should be large enough to cover the range of wavenumbers and frequencies where $U_{p, q}$ is significant. To avoid aliasing when sampling, suitable values of $\Delta \omega$ and $\Delta \xi$ are calculated with particular emphasis on satisfying Nyquist criterion. This is expressed mathematically as [17]

$$
\frac{1}{\Delta \omega} \geq\left|\frac{t}{\pi}\right|, \text { or }-\frac{\pi}{\Delta \omega} \leq t \leq \frac{\pi}{\Delta \omega} ; \text { and } \frac{1}{\Delta \xi} \geq\left|\frac{z}{\pi}\right| \text {, or }-\frac{\pi}{\Delta \xi} \leq z \leq \frac{\pi}{\Delta \xi}
$$

In order to explain how the computation of Eq. (21) is done, consider the mesh shown in Fig. 2. which is $2 \mathrm{D}$ projection of the picture in the $\xi-\omega$ domain. For any arbitrary point on the mesh $(p, q)$, the governing equation can be written as

$$
\begin{aligned}
& \left(E I \xi_{p}^{4}-m\left(\omega_{q}-\xi_{p} v\right)^{2}\right) U_{p, q}-D M \omega_{q}^{2} \sum_{r=-\alpha}^{\alpha} U_{r, q} \\
& +\sum_{s=-\beta}^{\beta} \sum_{r=-\alpha}^{\alpha}\left(\mathrm{i}\left(\omega_{s}-\xi_{r} v\right) C_{p-r, q-s}+K_{p-r, q-s}\right) U_{r, s}=P_{0} G_{p, q} .
\end{aligned}
$$

To avoid negative indices in the computation of Eq. (23), the extent of the mesh sizes for the coefficients $K_{d, e}$ and $C_{d, e}$, i.e. for $\tilde{\mathbf{k}}\left(\xi_{d}, \omega_{e}\right)$ and $\tilde{\mathbf{c}}\left(\xi_{d}, \omega_{e}\right)$, should be made twice the size of that required for $U_{p, q}$, i.e. for $\tilde{\mathbf{u}}\left(\xi_{p}, \omega_{q}\right)$. Therefore, $\sigma=2 \alpha$ and $\tau=2 \beta$. Also the summation indices are shifted from $(p=-\alpha, q=-\beta)$ to $(p=1, q=1)$ or in the case of $K_{d, e}$ and $C_{d, e}$ from $(d=-2 \alpha, e=-2 \beta)$ to $(d=1, e=1)$.

Let the number of sample points on the wavenumber and frequency domain be $N_{p}$ and $N_{q}$ respectively. The computation of Eq. (23) requires rearranging it into the form below

$$
\mathbf{H} \overline{\mathbf{U}}=\mathbf{P},
$$

where $\mathbf{H}$ is a matrix that contains the coefficients of $\overline{\mathbf{U}}$ and has size $N_{p} N_{q} \times N_{p} N_{q}, \overline{\mathbf{U}}$ and $\mathbf{P}$ are vectors of length $N_{p} N_{q}$, $\overline{\mathbf{U}}$ contains the displacement response in the $\xi-\omega$ domain whereas 
and $\mathbf{P}$ holds the external load. The elements of matrix $\mathbf{H}$ can be computed as follows

$$
\begin{aligned}
\mathbf{H}(g, h) & =\left(E I \xi_{\alpha+p+1}^{4}-m\left(\omega_{\beta+q+1}-\xi_{\alpha+p+1} v\right)^{2}\right) \\
& +\mathrm{i}\left(\omega_{\beta+s+1}-\xi_{\alpha+r+1} v\right) C_{2 \alpha+p-r+1,2 \beta-q+s+1} \\
& +K_{2 \alpha+p-r+1,2 \beta-q+s+1} ; \quad \text { for } r=p \text { and } s=q \\
\mathbf{H}(g, h) & =\left(E I \xi_{\alpha+p+1}^{4}-m\left(\omega_{\beta+q+1}-\xi_{\alpha+p+1} v\right)^{2}\right)-D M \omega_{\beta+q+1}^{2} \\
& +\mathrm{i}\left(\omega_{\beta+s+1}-\xi_{\alpha+r+1} v\right) C_{2 \alpha+p-r+1,2 \beta-q+s+1} \\
& +K_{2 \alpha+p-r+1,2 \beta-q+s+1} ; \quad \text { for all } r \text { and } b=q \\
\mathbf{H}(g, h) & =\mathrm{i}\left(\omega_{\beta+s+1}-\xi_{\alpha+r+1} v\right) C_{2 \alpha+p-r+1,2 \beta-q+s+1} \\
& +K_{2 \alpha+p-r+1,2 \beta-q+s+1} ; \quad \text { elsewhere }
\end{aligned}
$$

where $g$ and $h$ are indices obtained by repeating $\{-\alpha: \alpha\}$ and $\{-\beta: \beta\}^{T} N_{q}$ and $N_{p}$ times respectively. Note that the index $b$ is also the same as $s$ in this instance as they are both associated with frequency summations over the same interval. The force vector, $\mathbf{P}$, is composed of elements that are zero for $\left(\xi_{p}, \omega_{q} \neq \omega_{0}\right)$ and $\Delta \xi / 2 \pi$ for $\left(\xi_{p}, \omega_{q}=\omega_{0}\right)$.

Having obtained the response amplitudes, $U_{p, q}$, the response in the space-time domain is then calculated using Eq. (15).

\section{Application of the DFS method: step variation in foundation properties}

In this section, the DFS formulation is applied to a step variation in foundation stiffness and damping for continuously supported beams. A step variation in track foundation properties can occur in cases where a track section has been removed and replaced for maintenance purposes, and for which the resulting foundation has different stiffness and damping properties from the existing one surrounding the replaced section. Another practical case is when a different track design (e.g. floating slab) is adopted in one section of the track compared to the rest of the track. This can be done in order to reduce vibration levels near structures with more stringent vibration criteria.

Fig. 3 is a schematic diagram of a step variation in stiffness. In order to allow for smooth change in stiffness from one section to the next, two transition zones described by half-cycle cosine waves have been included. In Fig. 3 the subscripts ' $\mathrm{S}$ ' and ' $\mathrm{T}$ ' represent 'step' and

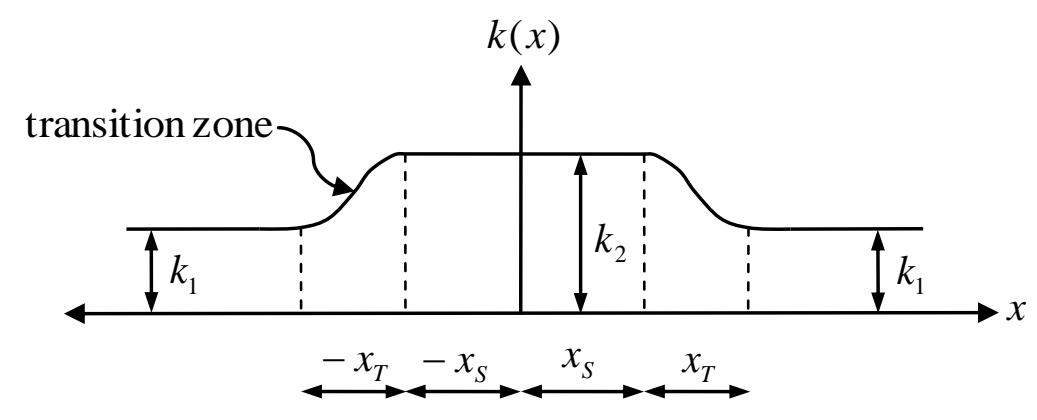

Figure 3: Variation of track foundation stiffness for continuously supported beam

'transition zone' respectively. It should be noted that $k_{2}$ has been symmetrically placed about the beam's origin merely for convenience and can be located anywhere along the beam length depending on the prevailing boundary conditions of one's problem. The stiffness, $k_{2}$, is related 
to $k_{1}$ through the nonhomogeneity parameter, $\mu$, such that $k_{2}=(1+\mu) k_{1}$, where $\mu$ is the ratio of the difference in stiffness, $\left(k_{2}-k_{1}\right)$, to the uniform stiffness, $k_{1}$.

The track foundation stiffness variation is described by

$$
k(x)= \begin{cases}k_{1} & \text { if }|x|>x_{S}+x_{T}, \\ k_{2} & \text { if }|x| \leqslant x_{S}, \\ k_{1}\left(1+0.5 \mu\left(1+\cos \left(\pi\left(x-x_{S}\right) / x_{T}\right)\right)\right) & \text { if } x_{S} \leqslant|x| \leqslant x_{S}+x_{T} .\end{cases}
$$

The foundation damping follows the same variation as in Fig. 3 with $c_{i}=2 \zeta \sqrt{\left(m k_{i}\right)}$; for $i=1,2$, where $\zeta$ is the damping ratio and has been assumed to have the same value for both sections. It follows therefore that $c_{2}=\sqrt{(1+\mu)} c_{1}$.

Now $k(x)$ and $c(x)$ should be transformed into $k(z, t)$ and $c(z, t)$ before being substituted into Eq. (5). In the fixed frame of reference, the foundation stiffness is independent of time and so the nonhomogeneous section is always at a constant position along the beam length (i.e. $\left.-x_{S}-x_{T}, x_{S}+x_{T}\right)$, see Fig. 4(a) for the case where $x_{T}=0$. In the moving frame of reference

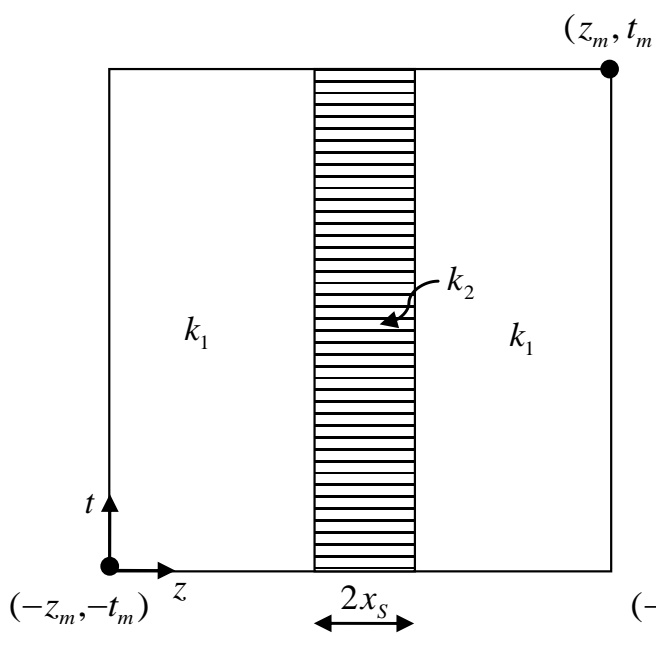

(a)

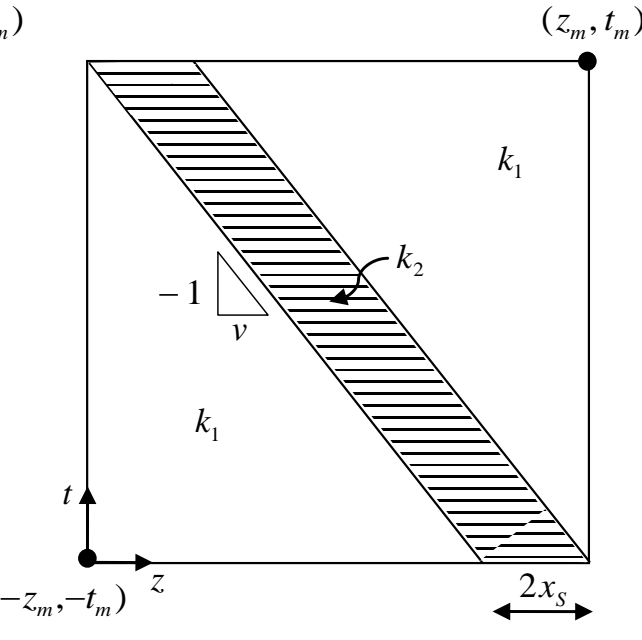

(b)

Figure 4: The foundation stiffness variation in (a) the fixed frame and (b) the moving frame of reference

in (b), on the other hand, the load 'perceives' the nonhomogeneous section to be at $+\infty$ as $t \rightarrow-\infty$. As the load moves, it gets closer to this section (at a constant rate of $-v$ ), until, at $t=0$, where the moving frame coincides the fixed frame. The load then moves to the opposite side of the section for positive values of $t$. The same analogy also applies for the foundation damping.

In section 5, results are presented for bean on elastic foundation with step variation in properties with and without transition zones.

\section{Results and discussions}

In this section, results are presented for the DFS method. Results are first presented in section (5.1) for the special case of a harmonic load moving along the beam, i.e. when $M=0$. Later in section (5.2), the effect of the presence of the mass is investigated. In each of these cases, the DFS method is compared with existing methods. Finally, convergence and computational considerations of the current method are discussed in section (5.3).

Table 1 contains the parameters used in the numerical examples presented in this section. 
Table 1: Parameters used in the numerical examples

\begin{tabular}{llr}
\hline Model components & Parameter & Parameter value \\
\hline \multirow{2}{*}{ Rail (60E1) } & Mass per unit length, $m$ & $60.21 \mathrm{~kg} \mathrm{~m}^{-1}$ \\
& Bending stiffness, $E I$ & $6.4 \mathrm{MN} \mathrm{m}^{2}$ \\
Railpad & Distributed stiffness, $k_{1}$ & $20 \mathrm{MN} \mathrm{m}^{-2}$ \\
& Damping ratio, $\zeta$ & 0.1 \\
Coupled mass & Mass magnitude, $M$ & $600 \mathrm{~kg}$ \\
& Force amplitude, $P_{0}$ & $1 \mathrm{~N}$ \\
\hline
\end{tabular}

\subsection{Results for the moving harmonic load}

\subsubsection{Validation of the DFS method}

Trochanis et al. [18] presented a unified procedure for analysing a beam on homogeneous elastic foundation subjected to an oscillating moving load. The method which is based on the Fourier Transformation Method (FTM) is applied to beams posed on Kelvin foundation. Like the current model, the analysis is carried out in the moving frame of reference. Using this method for the homogeneous case, the beam displacement response in the space-time domain can be computed from

$$
u(z, t)=\frac{P_{0} \mathrm{e}^{\mathrm{i} \omega_{0} t}}{2 \pi} \int_{-\infty}^{\infty} H\left(\xi, \omega_{0}\right) \mathrm{e}^{\mathrm{i} \xi z} \mathrm{~d} \xi,
$$

where $H\left(\xi, \omega_{0}\right)$ is the transfer function, given by

$$
H\left(\xi, \omega_{0}\right)=\frac{1}{E I \xi^{4}-m\left(\omega_{0}-\xi v\right)^{2}+\mathrm{i} c\left(\omega_{0}-\xi v\right)+k} .
$$

Equation (27) is computed numerically, adopting the same sampling considerations for the DFS method. This approach is used to check the validity of the results obtained from the DFS method with uniform stiffness and damping (i.e. for $\mu=0$ ).

Fig. 5(a-d) shows a comparison between the DFS and the FTM for the beam response in a moving frame of reference for selected load velocities and frequencies. In (a), (c) and (d), the beam displacement at $t=0$ for $f_{0}=10 \mathrm{~Hz}, v=36 \mathrm{kmh}^{-1}, f_{0}=100 \mathrm{~Hz}, v=180 \mathrm{kmh}^{-1}$ and $f_{0}=0 \mathrm{~Hz}, v=v_{c r}$ respectively are shown whereas (b) shows the displacement-time history under the moving load (i.e. $z=0$ ) for the same parameters as in (a). As can be seen from this figure, the agreement between the two methods is very good for all the cases shown. For low velocity and frequency, the beam displacement is symmetrical about the origin as is the dispersion curves and there are no propagating waves. This is because all the roots of the dispersion equation are imaginary and give rise to decaying solutions, see [19]. As the frequency and velocity increases, waves start to propagate from the load. For very low velocities $(v \rightarrow 0)$, the waves start propagating at the cut-on frequency, $f_{c o}=1 / 2 \pi \sqrt{\left(k_{1} / m\right)} \approx 92 \mathrm{~Hz}$. As the velocity increases, however, the cut-on frequency, $f_{c o} \rightarrow 0$. The velocity at which $f_{c o}=0 \mathrm{~Hz}$ is known as the critical velocity and is expressed, for Euler-Bernoulli beams, as $v_{c r}=\left(4 E I k_{1} / m^{2}\right)^{1 / 4}$. This gives $v_{c r}=613 \mathrm{~ms}^{-1}$ for the parameters in Table 1. At this velocity waves start to propagate from a static load (i.e. when $\omega_{0}=0$ ) as shown in (d).

\subsubsection{Step variation in foundation properties with no transition zones}

Fig. 6)(a-d) shows a comparison between the homogeneous and nonhomogeneous cases for $\mu=0$ and 0.25 respectively. The transition zone is non existent in this example, i.e. $x_{T}=0$ whereas $x_{S}=025 z_{m}$, for $z_{m}=10 \mathrm{~m}$ in (a) and (b) and $20 \mathrm{~m}$ in (c) and (d). The beam displacement response due to a load with $f_{0}=10 \mathrm{~Hz}$ and $v=36 \mathrm{kmh}^{-1}$ is shown in (a) at time $t=0$ 

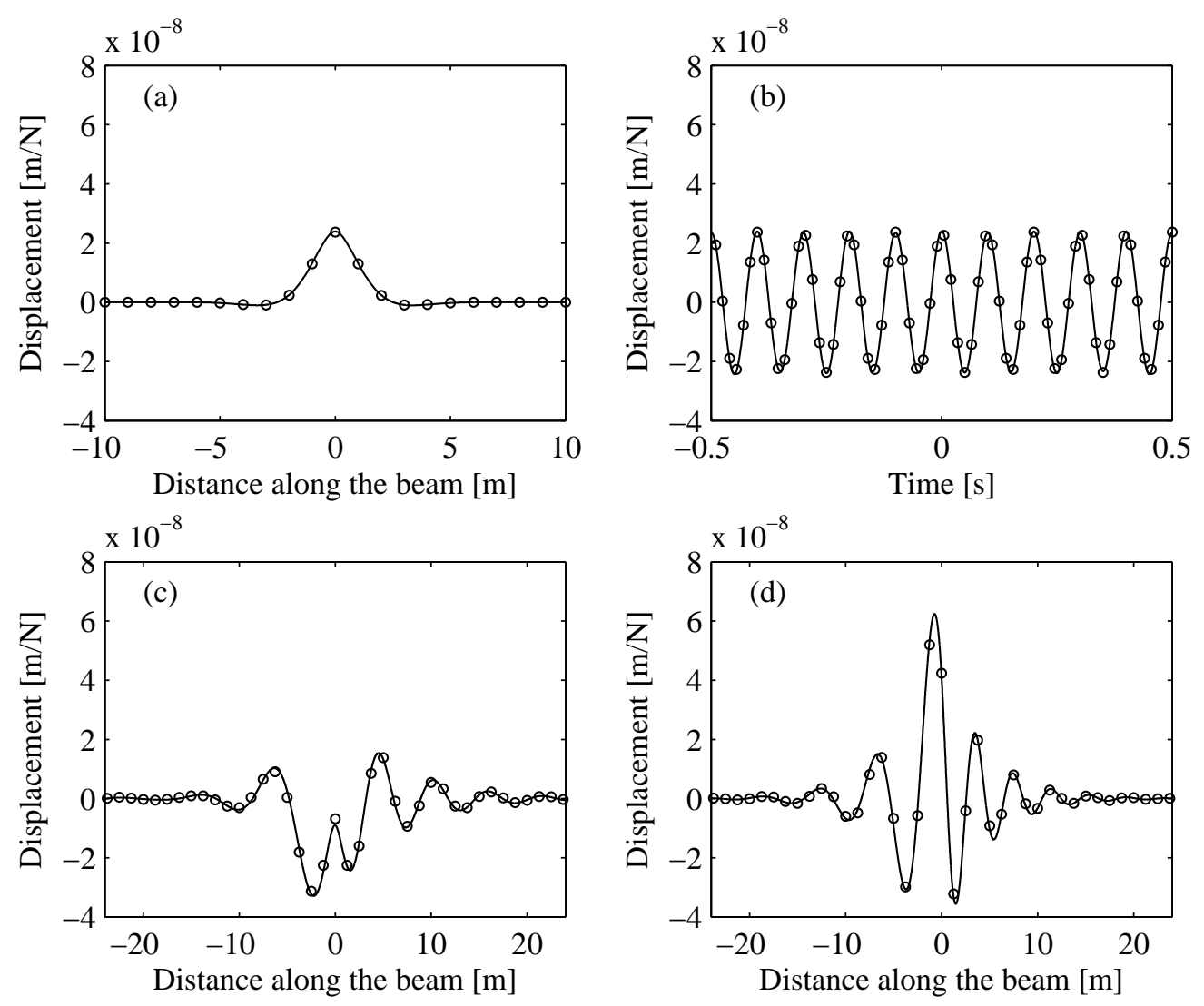

Figure 5: Beam displacement response in the moving frame of reference for various load velocities and frequencies; (a), (c) and (d), the beam displacement at $t=0$ for $f_{0}=10 \mathrm{~Hz}, v=36 \mathrm{kmh}^{-1}, f_{0}=100 \mathrm{~Hz}, v=180 \mathrm{kmh}^{-1}$, and $f_{0}=0 \mathrm{~Hz}, v=v_{c r}$ respectively, (b) displacement-time history under the moving load (i.e. $z=0$ ) for the same parameters as in (a). — : DFS, o: FTM

and in (b) for a point directly under the moving load, i.e. $z=0$. In (a), it is clear that the peak displacement amplitude of the beam is lower for the nonhomogeneous case than for the homogeneous case, due to the higher stiffness of $k_{2}$. This effect may however be considered to be local as the displacement amplitudes away from the region of $k_{2}$ converge for both cases. In (b) the response under the load is shown with the region of $k_{2}$ bounded within the vertical bold black lines. It can be seen that the steady state response is altered with the effect of increased stiffness within the region being a reduction in the amplitude of the response.

For the higher frequency case shown in (c), with $f_{0}=100 \mathrm{~Hz}$ and $v=80 \mathrm{kmh}^{-1}$, the nonhomogeneous case exhibits a larger peak response because the excitation frequency almost coincides with the resonance frequency for this case. This frequency can be seen in (d) to be around 100 $\mathrm{Hz}$ whereas the resonance frequency for the homogeneous case is at $92 \mathrm{~Hz}$. Observation of the results also shows that the responses are out of phase. The variations of the displacement at $z=0, t=0$ with frequency are shown in (d). The increased stiffness in the vicinity of $z=0$ leads to a reduction in the peak displacement of $1.8 \mathrm{~dB}$. This also leads to increased resonance frequency.

\subsubsection{Step variation in foundation properties with transition zones}

In this section the effect of step variation in foundation properties with transition zones is studied. The results obtained with the DFS method are compared with standard Finite Element Method (FEM) in the space-time domain, see [20, 21, 12]. For the FEM, the beam is discretised 

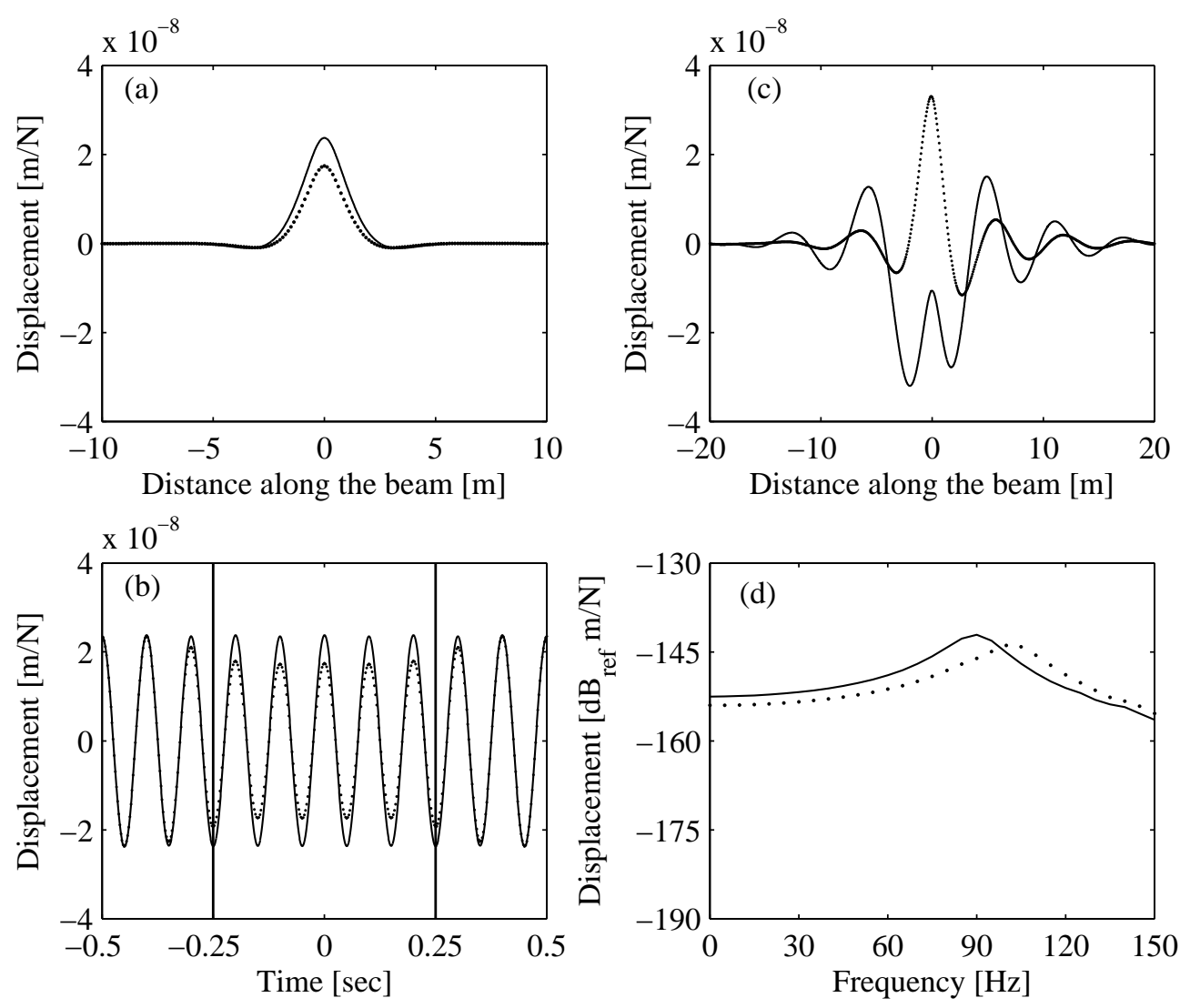

Figure 6: Beam displacements in the moving frame of reference for various load velocities and frequencies. (a) and (b) beam displacement at $t=0$ for $f_{0}=10 \mathrm{~Hz}, v=36 \mathrm{kmh}^{-1}$ and $f_{0}=100 \mathrm{~Hz}, v=80 \mathrm{kmh}^{-1}$ respectively, (c) displacement-time history under the moving load for the parameters in (a), and (d) beam displacement $u(0,0)$ plotted against oscillating frequency of a load moving at $36 \mathrm{kmh}^{-1} . \square^{\prime} \mu=0, \cdots: \mu=0.25$

into a finite number of two-noded Euler-Bernoulli beam elements, each of length, $L$, mass per unit length, $m$, and bending stiffness, EI. Each element has four degrees-of-freedom (dof), i.e. vertical translation and rotation at each node. The foundation stiffness and damping are respectively modelled with springs and dashpots placed at the vertical dofs of the nodes along the beam, with values derived from their distributed counterparts as $k L$ and $c L$ respectively. The global mass, $\mathbf{M}$, damping, $\mathbf{C}$, and stiffness, $\mathbf{K}$, matrices of the beam and elastic foundation as well as the equivalent nodal force vector, $\mathbf{P}$, are formed.

The dynamic behaviour of beam on elastic foundation under arbitrary loading conditions can be described by the time discretised differential equation

$$
\mathbf{M} \ddot{\mathbf{u}}+\mathbf{C} \dot{\mathbf{u}}+\mathbf{K u}=\mathbf{P} .
$$

For a moving harmonic load, the nodal force vector, $\mathbf{P}$, will have $\left\{-P_{0} \cos \left(\omega_{0} t-\varphi\right) N^{T}(x)\right\}$ as the only non-zero elements placed at the corresponding dofs of the element on which it acts, where $N(x)$ is the Hermitian shape functions, calculated for each position of the load, $(x, t)$. A phase shift, $\varphi$, is introduced to ensure that the load always arrives at the midpoint of the beam with a peak value. The solution of Eq. (29) for the displacement vector, $\mathbf{u}$, and its derivatives is obtained using a two sub-step composite implicit time integration scheme, see Bathe and Baig 22. In order to reduce boundary and transient effects, additional damping is provided at the first and last nodes of the beam. 
Figure 7 shows a comparison between the DFS method and FEM in the fixed frame of reference for beam resting on an elastic foundation with step variation properties with $x_{S}=x_{T}=3$ $\mathrm{m}$ and $\mu=0.5$. The spatial variation in stiffness is given in (a). In (b), the displacement under the moving load for $f_{0}=30 \mathrm{~Hz}, v=160 \mathrm{kmh}^{-1}$ is shown whereas (c) shows the displacementtime history for a point at $x=0$ on the beam. For the DFS, $\Delta z=0.25, \alpha=60$ and $\beta=10$, giving a total beam length of $30 \mathrm{~m}$. Once matrix $\mathbf{H}$ has been computed, the response in the space-time domain is obtained with a much finer mesh, namely $\Delta z / N$, where $N$ is an integer. For the FEM, on the other hand, the beam is discretised into $120,0.25 \mathrm{~m}$ elements, i.e. $30 \mathrm{~m}$ length of beam. The time step used is $\Delta t=L / N v$, with $N$ taken as 10 for both methods.
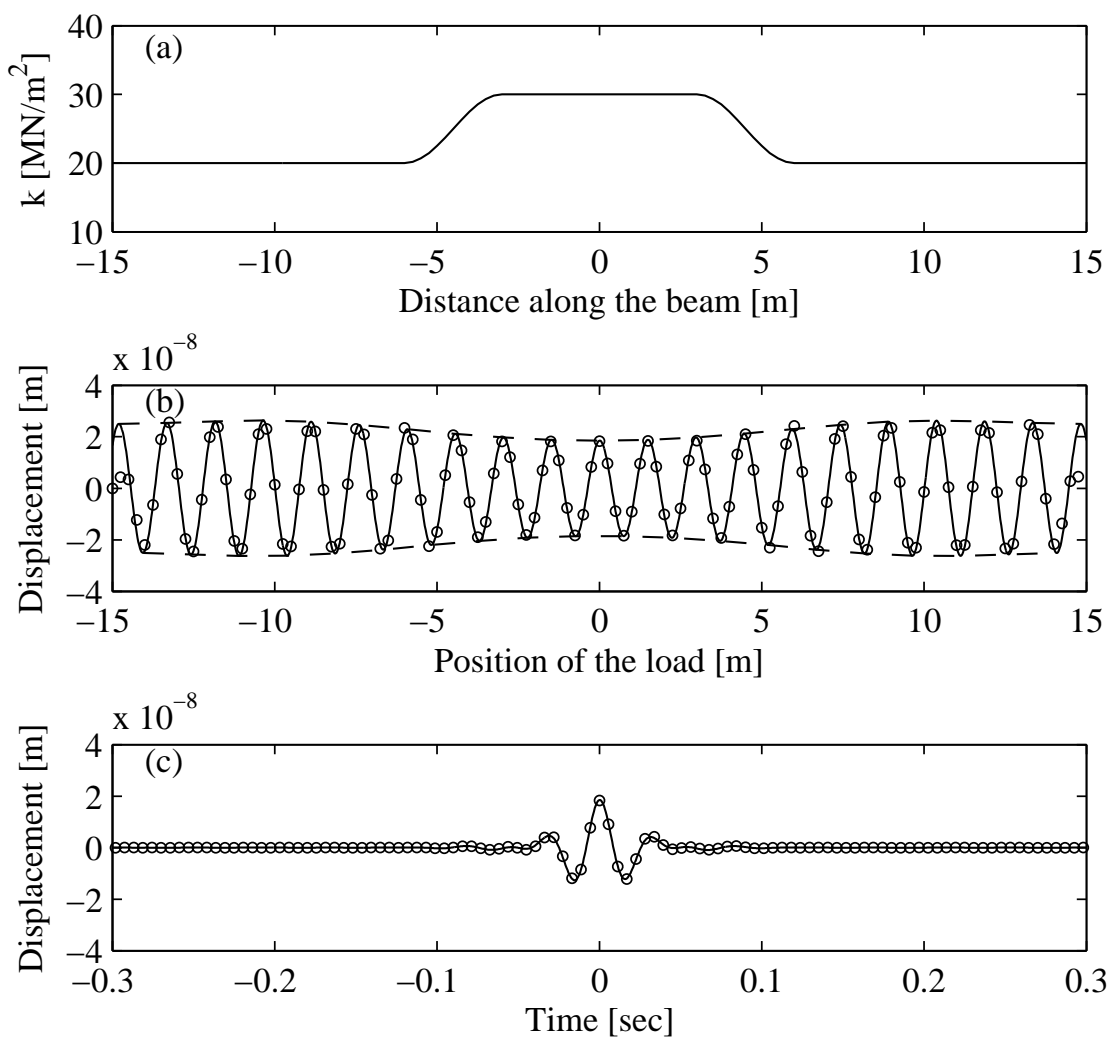

Figure 7: (a) Spatial variation of the foundation stiffness used in the simulation, (b) displacement under the moving load for $f_{0}=30 \mathrm{~Hz}, v=160 \mathrm{kmh}^{-1}$, displacement-time history in the fixed frame of reference of a point at $x=0$. — : DFS; ○: FEM; - - : displacement envelope

The results obtained from the two methods display very good agreement, although the FEM is computationally more efficient in terms of running time and memory usage. These simulations were carried out on an HP Pavilion computer, model p6-2000ukm, with Intel Core i3 processor @ $3.3 \mathrm{GHz}$ and $8 \mathrm{~GB}$ of RAM. The FEM runs in 1.2 seconds utilising $680 \mathrm{MB}$ of memory, whereas the DFS runs in 12.24 seconds utilising $774 \mathrm{MB}$ of memory. Although the system matrices in both cases need only be inverted once, the size of the matrix in the FEM is considerably smaller than that of the DFS method, hence the faster running time.

The effect of the transition zones is apparent in (b) as a clear envelope identical to the variation shown in (a) can be observed. As the load moves along the beam, it perceives an effective stiffness and damping underneath it. These effective properties can be considered local to the load - at $\sim 3-4 \mathrm{~m}$ - on either side of the load. At $x \rightarrow-\infty$, far away from the nonhomogeneous section, the effective stiffness and damping have constant values of $k_{1}$ and $c_{1}$ respectively and the load exhibits a steady state vibration with the largest response amplitude. As the load approaches the vicinity of the nonhomogeneous section, the stiffness and damping increase 
steadily with a corresponding steady reduction in the response amplitude and the steady state vibration is altered. The smallest response amplitude is observed around $x=0$, as the effective stiffness is largest. The reverse happens for $x>0$ as the load leaves this section.

\subsubsection{Effects of the length and magnitude of inhomogeneity}

Fig. 8 shows the effects of the length and magnitude of the inhomogeneity on the beam displacement, $u(0,0)$, for $f_{0}=10 \mathrm{~Hz}, v=36 \mathrm{kmh}^{-1}$. In (a) no transition zones are present where as in (b) transition zones are included. In (a), the homogeneous foundation case can be seen for two instances; i.e. when $\mu=0$ and when $x_{S}=0$. All other combinations of $\mu$ and $x_{S}$ depict the nonhomogeneous case. For each value of $\mu$, the amplitude of the response reduces with an increase in $x_{S}$, as the effective stiffness around $z=0$ increases. There is a rapid
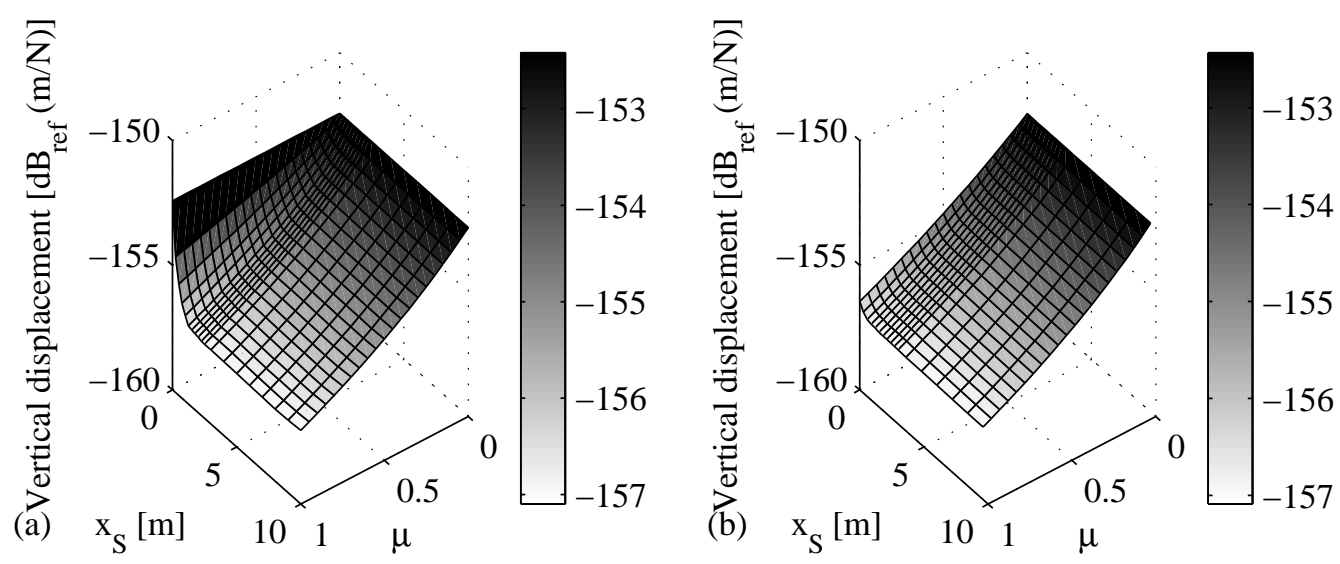

Figure 8: The effects of length and magnitude of the inhomogeneity on the response of a continuously supported beam $u(0,0)$ due to a load with $f_{0}=10 \mathrm{~Hz}, v=36 \mathrm{kmh}^{-1}$; (a) without transition zones (i.e. $x_{T}=0$ ) and (b) with transition zones (i.e. $x_{T}=2 \mathrm{~m}$ ).

reduction in the displacement of up to $4.6 \mathrm{~dB}$ from $x_{S}=0$ to $x_{S}=4 \mathrm{~m}$, after which point the influence of $k_{2}$ on $u(0,0)$ becomes insignificantly small as $u(0,0)$ changes only slightly by up to $0.1 \mathrm{~dB}$. This is because the effect $k_{2}$ on $u(0,0)$ is local to the vicinity of $z=0$. As $x_{S}$ becomes larger, the effective stiffness around $z=0$ converges to $k_{2}$ and the response amplitude $u(0,0)$ for the nonhomogeneous case also converges to that of the homogeneous case with stiffness and damping values corresponding to $k_{2}$ and $c_{2}$ respectively. It can also be seen that as $\mu$ increases, the displacement amplitude reduces due to the increase stiffness induced by the step change. In (b), the homogeneous case is obtained only for $\mu=0$. When $x_{S}=0$, there is a full-cycle cosine wave inhomogeneity from $\left\{-x_{T}: x_{T}\right\}$. This is why the response amplitude is not constant but reduces with increase in $\mu$ values. Again, the effect of $k_{2}$ can be seen to be local to the vicinity of $z=0$ and similar analogy as in (a) also applies here.

\subsection{Results for the moving harmonically excited mass}

In this section, results are presented to study the dynamic behaviour of a mass moving on a beam on nonhomogeneous elastic foundation and subjected to harmonic excitation. Comparisons of the DFS method with standard FEM in the space-time are carried out. The FEM is the same as that described in section (5.1.3). However, the presence of the coupled mass results in time-dependent system matrices due to its changing position as it moves along the beam.

The dynamic behaviour of a mass, $M$, moving on a beam on elastic foundation at a constant speed, $v$, and excited by an arbitrary force can be described by the time discretised differential 
equation

$$
\begin{aligned}
& {\left[\mathbf{M}+M\left[N^{T}(x) N(x)\right]\right] \ddot{\mathbf{u}}+\left[\mathbf{C}+2 v M\left[N^{T}(x) N^{\prime}(x)\right]\right] \dot{\mathbf{u}}} \\
& +\left[\mathbf{K}+v^{2} M\left[N^{T}(x) N^{\prime \prime}(x)\right]\right] \mathbf{u}=\mathbf{P} .
\end{aligned}
$$

The global nodal force vector, $\mathbf{P}$, has the same description as before. Similarly, the solution procedure for the displacement vector, $\mathbf{u}$, and its derivatives remains the same.

\subsubsection{Comparison between the DFS and FEM for the homogeneous case}

In this section, the results obtained for a harmonically excited mass moving on the beam with homogeneous foundation parameters are presented. The same discretisation parameters used for Fig. 7 in section (5.1.3) are also used here in order to compare the computational efficiency of both methods.

Figure $9(a-b)$ shows the beam displacement response in the fixed frame of reference due to a unit harmonic load superimposed on the moving mass for $f_{0}=40 \mathrm{~Hz}, v=250 \mathrm{kmh}^{-1}$. Both methods match very closely, although the transient state in the FEM solution results in
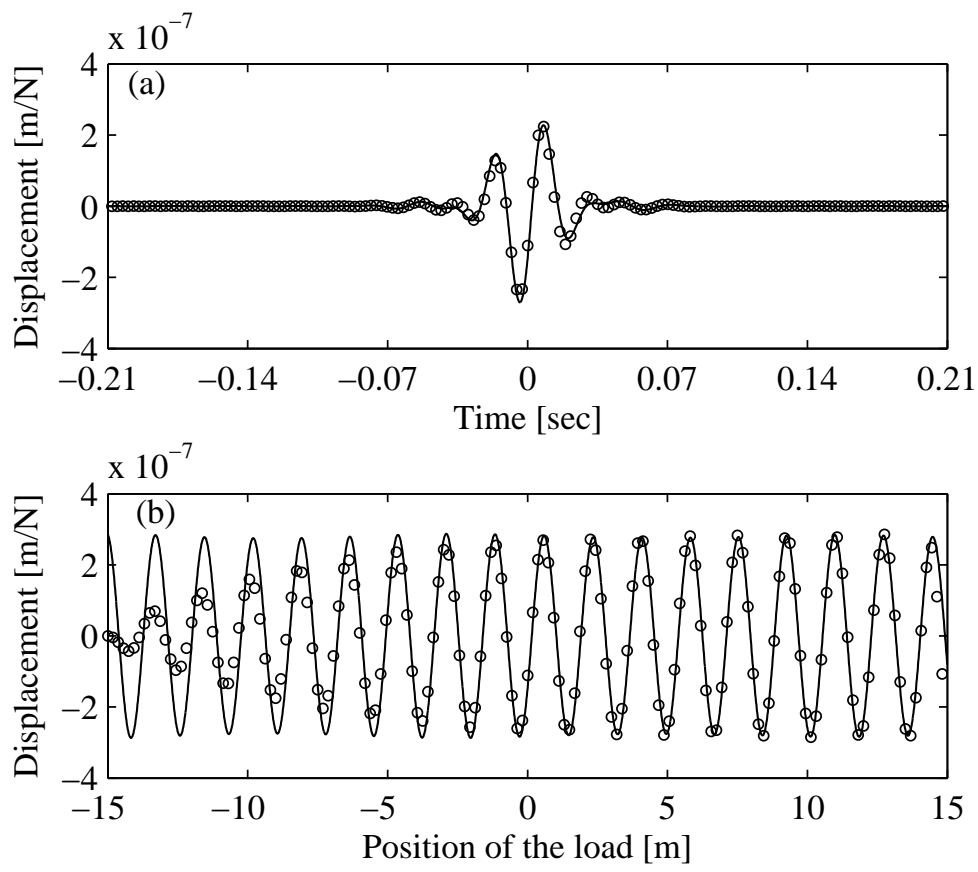

Figure 9: Beam displacement response in the fixed frame of reference for a mass with superimposed load oscillating with $f_{0}=40 \mathrm{~Hz}, v=250 \mathrm{kmh}^{-1}$, (a) displacement-time history at $x=0$, (b) displacement under the load. — DFS; ०: FEM.

a slight phase difference from the steady state DFS solution. The computation time of the FEM drastically increases from 1.2 seconds for the moving harmonic load case to 7.5 seconds in this. This is because the system matrices are now time dependent and need to be updated and inverted at each time step. For the DFS, on the other hand, the computing time only increases from 12.24 seconds to 13.9 seconds, since the matrix, $\mathbf{H}$, still needs to be formed and inverted only once. This is still longer than the FEM. However, numerical experiments show that by doubling the value of $N$ from 10 to 20, the computing time for the FEM also doubles to 15.17 seconds whereas the DFS only increases to 14.6 seconds. Therefore, for cases where very small time steps are required, it may be advantageous to adopt the DFS method instead of the FEM.

Figure 10 shows the maximum beam displacement at $x=$, plotted as a function of oscillating frequency of a harmonic load superimposed on a mass moving at $v=36 \mathrm{kmh}^{-1}$. The beam is 


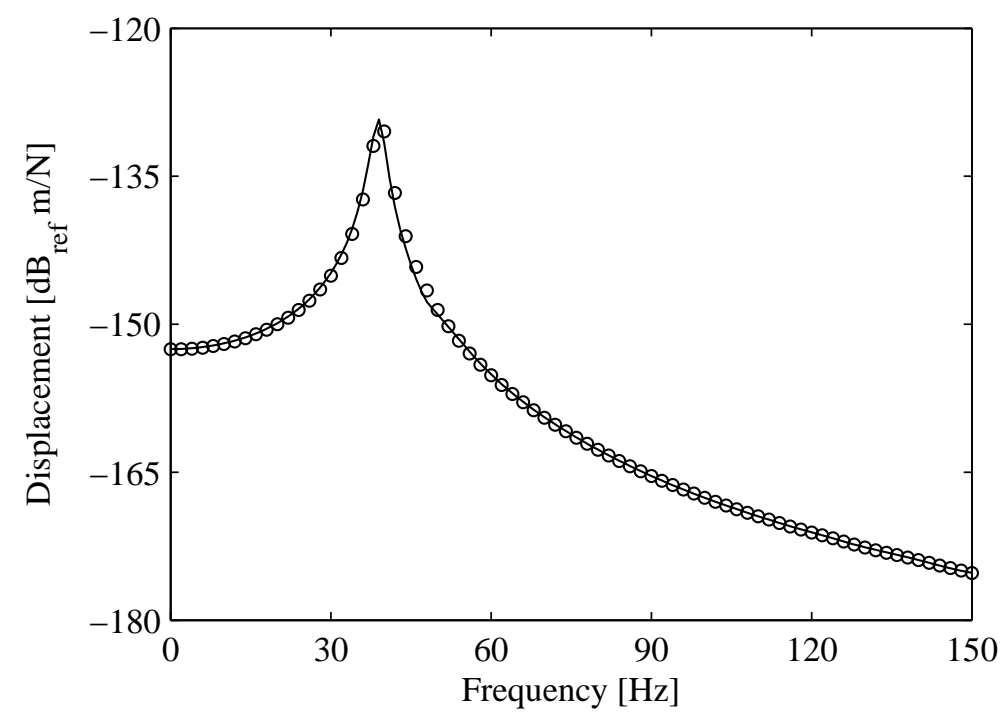

Figure 10: Maximum beam displacement at a point at $x=0$ plotted against frequency for a mass with superimposed oscillating load moving with $v=10 \mathrm{~ms}^{-1}$. Comparison between DFS ( - ) and FEM (o)

$40 \mathrm{~m}$ long and a frequency step of $1 \mathrm{~Hz}$ has been used. Comparison is also done between the DFS method and FEM for the homogeneous case. Unlike the moving harmonic load case where the beam resonance occurs at the $\omega_{c o}=\sqrt{k / m}$, the presence of the moving mass lowers the mass-beam resonance to a frequency, $\bar{\omega}_{c o}$, that can be estimated by finding the roots of [17]

$$
\frac{M^{4}}{64 E I} \bar{\omega}_{c o}^{8}+\left(m \bar{\omega}_{c o}^{2}-k_{e q}\right)^{3}=0,
$$

where $k_{e q}$ is the equivalent foundation stiffness; $k_{e q}=k_{1}$ for $\mu=0$ and $k_{1} \leq k_{e q} \leq k_{2}$ for $\mu \neq 0$. For the current set of parameters, $\bar{f}_{c o}=\bar{\omega}_{c o} / 2 \pi \approx 39 \mathrm{~Hz}$ for the case of $\mu=0$ which corresponds to the resonance frequency in the figure. Although Eq. (31) is only valid for a stationary mass, it can also be used to approximate the resonance frequency for the case of a mass moving with a velocity that is by far smaller than the critical velocity.

\subsubsection{Comparison between the DFS and FEM for the nonhomogeneous case}

Results are now presented to show the effect of the mass on the beam with nonhomogeneous foundation properties. Figure 11(a-b) shows the beam displacement response in the fixed frame of reference due to a unit harmonic load superimposed on the moving mass for $f_{0}=40 \mathrm{~Hz}$, $v=250 \mathrm{kmh}^{-1}$. The step variation parameters used in Fig. 7 of section (5.1.3) are also utilised for this example. Since the excitation frequency is greater than the cut-on frequency of the mass-beam system, propagating waves exist in the beam. This is evident from the lack of symmetry in both the point response and that under the load, as more waves propagate behind the load than ahead of it. This wave convection results in a shift in the displacements corresponding to the step change in stiffness.

Figure 12 shows the maximum beam displacement at $x=0$, plotted as a function of oscillating frequency of a harmonic load superimposed on a mass moving at $36 \mathrm{kmh}^{-1}$. Again, the beam is $40 \mathrm{~m}$ long and a frequency step of $1 \mathrm{~Hz}$ has been used for the DFS method. The effect of increasing and decreasing the stiffness is investigated with $\mu=-0.5$ and 0.5 . The homogeneous case has also been shown for comparison. It is apparent that the change in stiffness in the vicinity of $k_{2}$ increases the peak response amplitude when $k_{2}<k_{1}$ and decreases it when $k_{2}>k_{1}$. These also lead to accompanying reduction and increase in the resonance frequency 

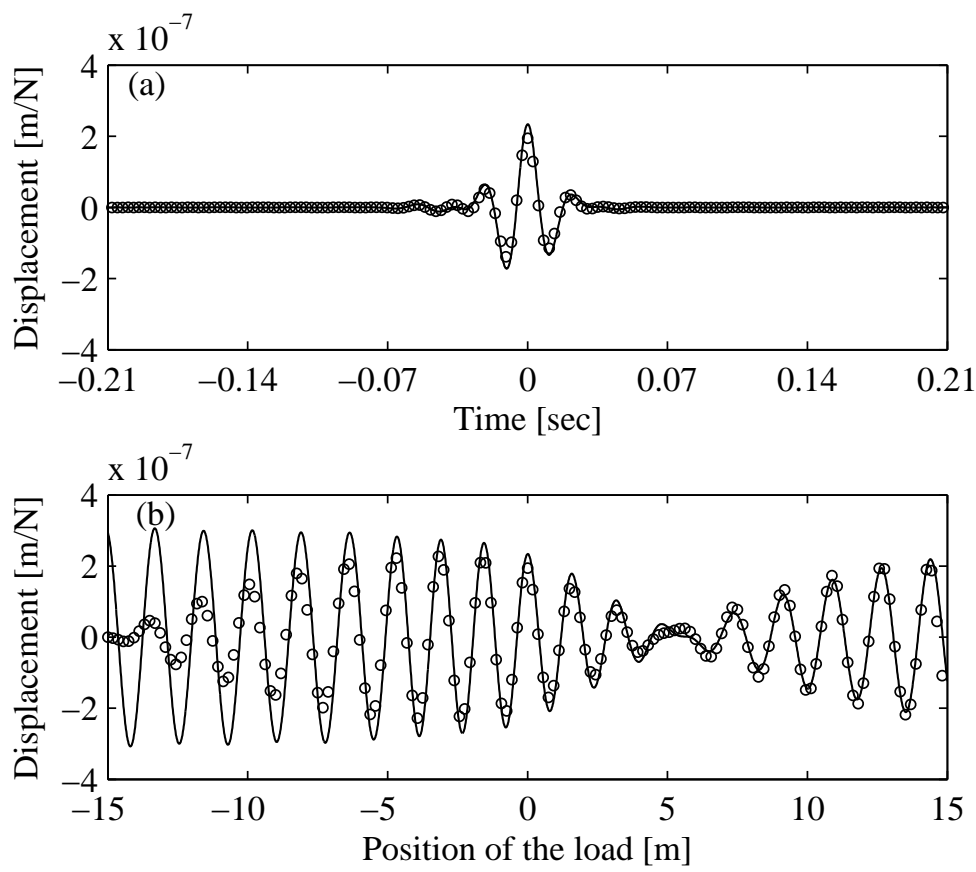

Figure 11: Beam displacement response in the fixed frame of reference for a mass with superimposed load oscillating with $f_{0}=40 \mathrm{~Hz}, v=250 \mathrm{kmh}^{-1}$, (a) displacement-time history at $x=0$, (b) displacement under the load. — : DFS; ○: FEM.
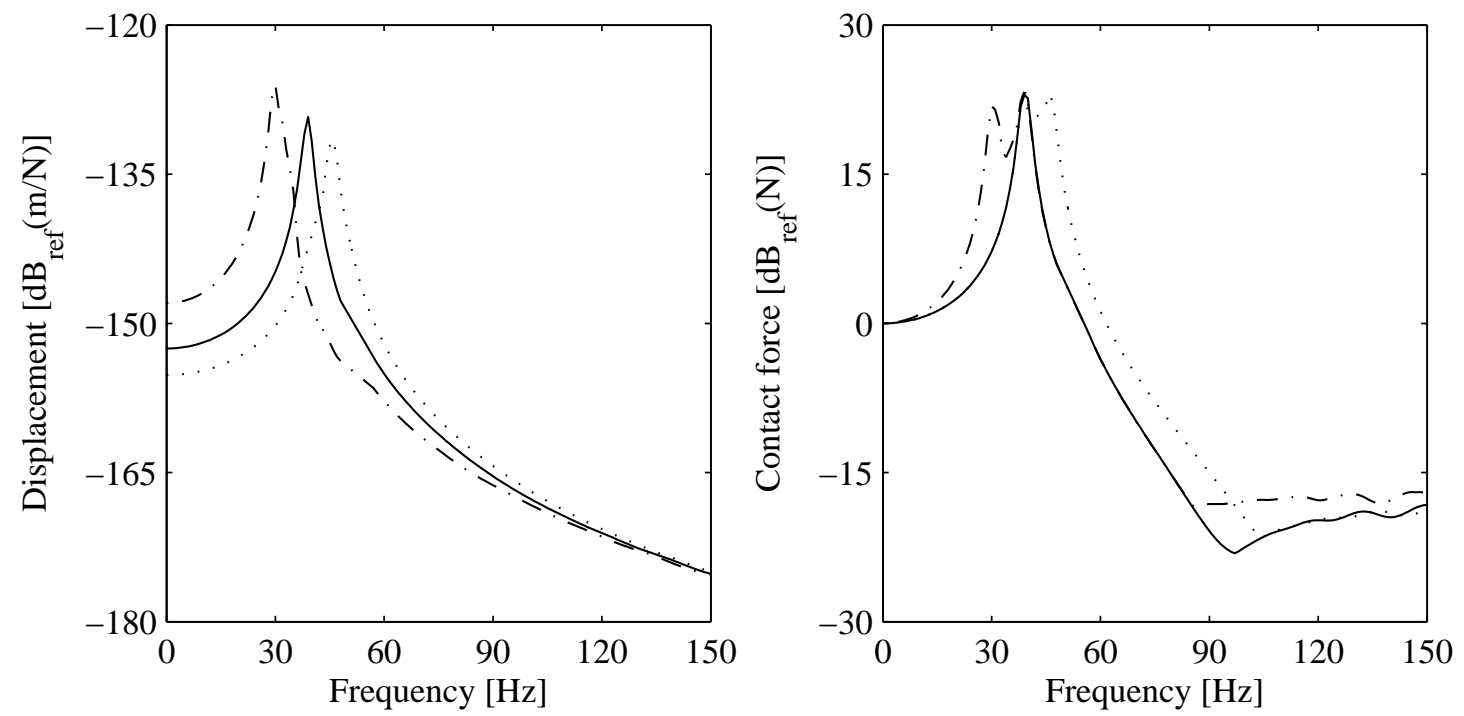

Figure 12: (a) Maximum beam displacement at a point at $x=0$ plotted against frequency for a mass with superimposed oscillating load moving with $v=10 \mathrm{~ms}^{-1}$, (b) corresponding contact force at the mass-beam interface. $\longrightarrow: \mu=0,-\cdot-: \mu=-0.5, \cdots \cdots: \mu=0.5$

respectively. However, the effect of decreasing stiffness is not the same as that of increasing it. For example, the peak displacement amplitude at the respective resonances increase by 4.9 $\mathrm{dB}$ when $k_{2}=0.5 k_{1}$ but only decrease by $2.4 \mathrm{~dB}$ when $k_{2}=1.5 k_{1}$. The resonance frequencies can be calculated using Eq. (31), in which $k_{e q} \approx k_{2}$, for the nonhomogeneous cases. This gives values of $30 \mathrm{~Hz}$ and $46 \mathrm{~Hz}$ for $\mu=-0.5$ and 0.5 respectively.

The corresponding contact force at the mass-beam interface is plotted versus frequency in Fig. 
12(b). The contact force is calculated in accordance with Eq. (2), for $x=v t$. Unlike the homogeneous case where only one resonance occurs, two distinct resonance peaks exist for the nonhomogeneous cases. Based on the oscillating frequency of the load, maximum contact force can be exhibited at different sections along the beam, due to the different stiffness, and hence different resonance frequencies. One of these peaks occurs when the oscillating frequency of the load coincides with the resonance corresponding to foundation stiffness, $k_{1}$, and occurs when the mass is far away from the region of $k_{2}$. The other peak occurs when the load's frequency matches that corresponding resonance frequency of the mass/beam on equivalent stiffness, $k_{1}<k_{e q} \leq k_{2}$, and occurs when the load is within the region $k_{2}$. Note that when $x_{S}$ is long enough, as is in this case, $k_{e q}=k_{2}$ around $x=0$, so that this resonance frequency will be the same as that corresponding to the mass/beam vibrating on $k_{2}$.

\subsubsection{Effect of mass magnitude and level of inhomogeneity}

The effect of the magnitude of a coupled mass on the dynamic behaviour of the beam on elastic foundation with step variation in properties is analysed in this section. Figure 13 shows the variation of the peak displacements at resonance for a point at $x=0$, plotted as a function of $M$ and $\mu$. The mass magnitudes range from 0 to $1000 \mathrm{~kg}$ with a step of $100 \mathrm{~kg}$ whereas $\mu$ ranges from -0.75 to 0.75 with a step of 0.25 . The length of the beam is $40 \mathrm{~m}$, with $x_{S}=x_{T}=5$ $\mathrm{m}$ and the speed of the mass is $36 \mathrm{kmh}^{-1}$.

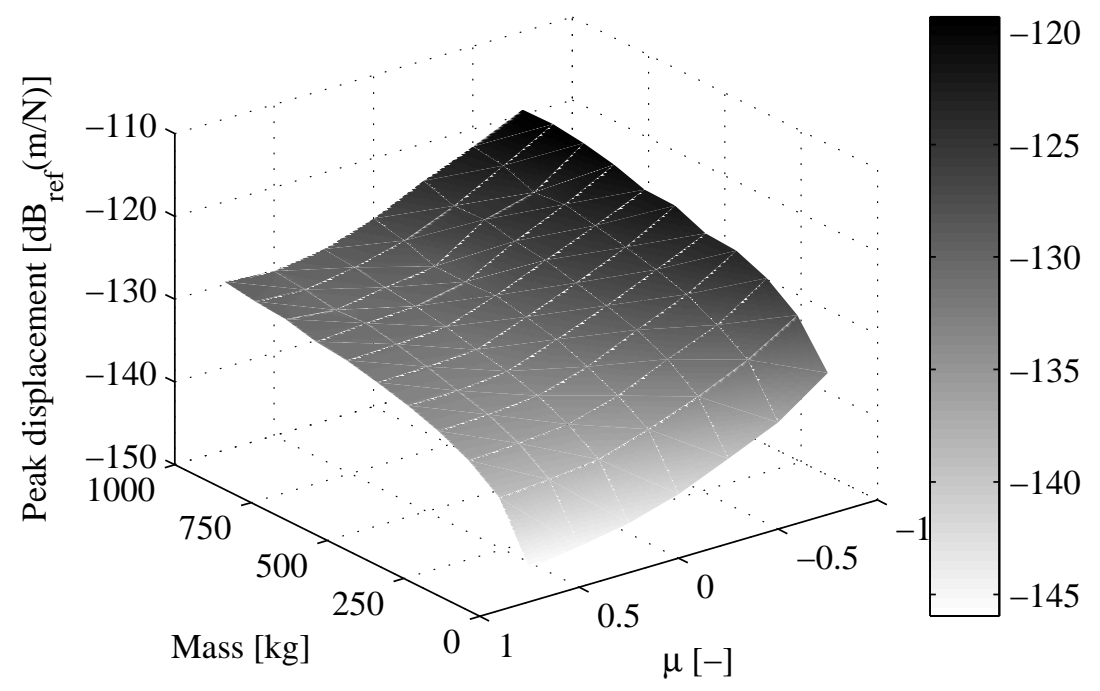

Figure 13: The effect of magnitude of the mass and degree of inhomogeneity on the resonant displacement amplitude of the beam due to a load moving at $v=36 \mathrm{kmh}^{-1}$.

The effect of increasing the mass magnitude is an almost hyperbolic increase in the resonance displacement amplitude. For the range of mass shown, the peak displacement increases by up to $13.5 \mathrm{~dB}$ when $k_{2}$ is at its lowest value and by $16.4 \mathrm{~dB}$ when $k_{2}$ is largest. Also, the effect of increasing the foundation stiffness over the range of $\mu$ is a reduction in the peak displacement amplitudes by $13.1 \mathrm{~dB}$ for the moving harmonic load and $10.2 \mathrm{~dB}$ for the heaviest moving mass.

\subsection{Convergence and computational considerations of the DFS method}

\subsubsection{Convergence}

Two fundamental parameters are of interest when using the DFS method; i.e. $\Delta z$ and $\Delta t$. The other computational parameters are derived from these in order to satisfy Nyquist sampling criteria and the prevailing boundary conditions at $z= \pm \infty$. For example, $\omega_{m}$ and $\xi_{m}$ 
are defined as $\pi / \Delta z$ and $\pi / \Delta t$ respectively where as $z_{m}$ and $t_{m}$ are controlled by the load velocity and excitation frequency and are chosen large enough to ensure that the boundary conditions are satisfied. Similarly, $\alpha=z_{m} / \Delta z$ and $\beta=t_{m} / \Delta t$. For frequencies sufficiently lower than the cut-on frequency $f_{c o}$ and velocities well below the critical velocity $v_{c r}$, the boundary conditions are generally satisfied for $z_{m}=8 \sim 12 \mathrm{~m}$ as there are no propagating waves. At frequencies higher than $f_{c o}$ on the other hand, propagating waves exist and the boundary conditions are only satisfied at larger values $z_{m}$. It follows therefore that convergence study of the DFS method is most important for these two parameters. Fig. 14 shows the convergence analyses
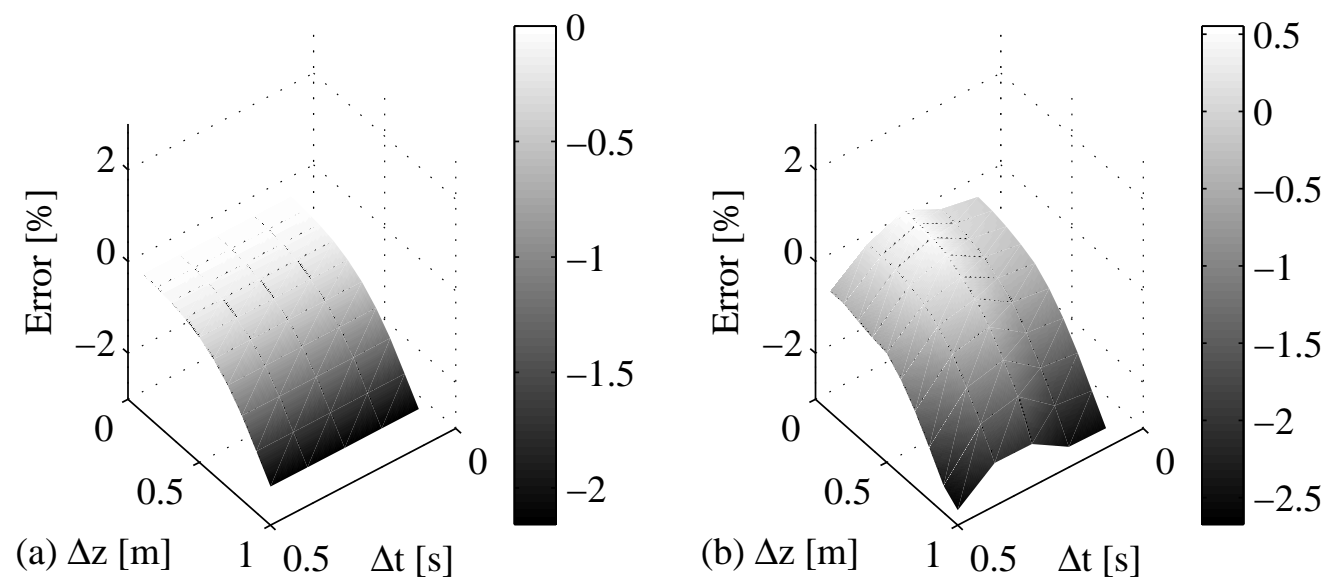

Figure 14: Convergence of the DFS method for various values of $\Delta z$ and $\Delta t$ for a harmonic load moving on the beam with $f_{0}=10 \mathrm{~Hz}, v=36 \mathrm{kmh}^{-1}$ (a) the homogeneous case, (b) the nonhomogeneous case with $\mu=0.25$, $x_{S}=4 \mathrm{~m}$ and $x_{T}=0$

of the beam response on $\Delta z$ and $\Delta t$ for $f_{0}=10 \mathrm{~Hz}, v=36 \mathrm{kmh}^{-1}$ in terms of percentage errors from the converged value. Fig. 14(a) depicts the homogeneous case whereas (b) the nonhomogeneous case. In (a) it can be seen that the error increases as the spatial step size $\Delta z$ increases. However, no dependence of the response on $\Delta t$ can be observed. This is because, for the homogeneous case, the foundation properties are constant and therefore independent of time. In the nonhomogeneous case in (b), on the other hand, the foundation properties change both with time and space. When $\Delta z$ is small, the step inhomogeneity is well defined. Also when $\Delta t$ is small, it ensures that the load spends enough time in the vicinity of the inhomogeneity to capture the true dynamic response of the beam. For large values of $\Delta z$ and $\Delta t$, however, the step inhomogeneity is ill defined and the load passes the section very quickly. Hence the highest absolute errors of up to $2.67 \%$ can be observed in these instances.

\subsubsection{Computational considerations}

Using the DFS method can present some challenges in terms to computing limitations (namely, RAM usage in MATLAB) because of the large coefficient matrix that needs to be formed and inverted to obtain the solution. The maximum mesh sizes (i.e. in terms of $\Delta z, \Delta t, \alpha$ and $\beta$ ) that can be accommodated to give converged results can be restricted. This is particularly true for high frequency, high velocity cases where the bending waves in the beam propagate to infinitely long distances along the beam length and therefore $z_{m}$ should be large enough to satisfy the boundary conditions. For these cases, the time and spatial step sizes need to be small thus resulting in large sampling frequencies and wavenumbers. However, the size of the mesh is restricted in terms of $\alpha$ and $\beta$ values, so one ends up with very large $\Delta \xi$ and $\Delta \omega$.

Examples of computational values used to obtain the results presented in the preceding sections are given below. For frequencies sufficiently lower than $f_{c o}$ and velocities well below $v_{c r}$, the 
mesh size is small enough to be easily computed with $\Delta z=0.25 \sim 0.5 \mathrm{~m}, \Delta t=0.1 \sim 0.2 \mathrm{~s}$, $\alpha=30 \sim 50$ and $\beta=10 \sim 15$. For high frequency, high velocity cases, however, the mesh size may be large with $\Delta z=0.15 \sim 0.25 \mathrm{~m}, \Delta t=0.05 \sim 0.1 \mathrm{~s}, \alpha \geq 80$ and $\beta \geq 10$. These parameters have proven to be sufficient as the DFS method compares well with other methods presented earlier. Two suggestions on managing memory usage and mesh sizes are given below

1. Since the majority of the memory requirement is dominated by the formation and inversion of the coefficient matrix $\mathbf{H}$, memory can be 'freed up' by clearing $\mathbf{H}$ once the vector $\overline{\mathbf{U}}$ has been computed as the response in the space-time domain is only a function of the latter.

2. A coarse mesh may be used to obtain the wavenumber-frequency domain solution without compromising the accuracy of the results. This is because most of significant values of the response are concentrated only within a small range of frequency and wavenumber. After this is done, very fine mesh sizes are then used to obtain the space-time domain responses.

With the ever growing developments in computing capabilities, such as high performance computing, however, all these limitations can be easily exceeded.

\section{Conclusions}

A new method has been presented for analysing the vibration behaviour of a beam continuously supported on a linear nonhomogeneous elastic foundation under the action of a harmonically excited mass. The method involves the decomposition of the beam displacement response and the spatially varying foundation stiffness and damping into Double Fourier Summations (DFS), which are solved numerically to give the response in the wavenumber-frequency domain. The corresponding response in the space-time domain is then obtained by back substitution into the DFS expression of the response. The method has been applied to step variation in foundation properties with and without transition zones, and its validity has been checked against existing techniques. Some general conclusions derived from this work are summarised below

- The DFS portrays excellent agreement with existing methods for solving the problem of a beam on elastic foundation with both constant and varying parameters under the action of a moving load and mass.

- The DFS is not restricted to small variations in foundation properties as does the first order perturbation technique which has been widely used for such problems. This means that even larger degrees of inhomogeneity can be analysed with the DFS formulation. This is because any variation of the nonhomogeneous parameters in the space-time domain can be decomposed into its harmonic components in the DFS form. Therefore, it is paramount that the sampling is done in the correct manner in order to obtain accurate results.

- For a harmonically excited mass moving with low speeds, the effects of inhomogeneity in foundation stiffness can be summarised as a reduction in the amplitude of the response in the neighbourhood of the change in stiffness and an increase in the resonance frequency of the beam. This is only true for $\mu>0$, with the reverse occurring for $\mu<0$.

- A beam on nonhomogeneous elastic foundation may exhibit multiple resonances corresponding to the foundation stiffness of individual sections. Although this is not evident in point displacements, it is clear from the displacement, and hence the contact force at the mass/beam interface as the mass moves through the respective sections along the beam. 


\section{Acknowledgements}

The authors wish to acknowledge the funding of this work through the Dean of Engineering Research Scholarship for International Excellence at the University of Nottingham.

\section{References}

[1] L. Fryba. Vibration of Solids and Structures under Moving Loads, volume 3rd. Thomas Telford, London, 1999.

[2] A.K. Mallik, S. Chandra, and A.B. Singh. Steady-state response of an elastically supported infinite beam to a moving load. Journal of Sound and Vibration, 291:1148-1169, 2006.

[3] A.V. Metrikine and K. Popp. Steady-state vibrations of an elastic beam on a visco-elastic layer under moving load. Archive of Applied Mechanics, 70:399-408, 2000.

[4] H. Hunt. Types of rail roughness and the selection of vibration isolation measures. In Noise and Vibration Mitigation for Rail Transportation Systems, volume 99, pages 341-347, Berlin, 2008. Springer Berlin / Heidelberg.

[5] A.A. Mahmoud and M.A. El Tawil. Beams on random elastic supports. Applied Mathematical Modelling, $16(6): 330-334,1992$.

[6] L. Frýba, S. Nakagiri, and N. Yoshikawa. Stochastic finite elements for a beam on a random foundation with uncertain damping under a moving force. Journal of Sound and Vibration, 163(1):31-45, 1993.

[7] L. Andersen and S.R.K. Nielsen. Vibrations of a track caused by variation of the foundation stiffness. Probabilistic Engineering Mechanics, 18(2):171-184, 2003.

[8] S.N. Verichev and A.V. Metrikine. Instability of vibrations of a mass that moves uniformly along a beam on a periodically inhomogeneous foundation. Journal of Sound and Vibration, 260(5):901-925, 2003.

[9] M.N. Pavlović and G.B. Wylie. Vibration of beams on non-homogeneous elastic foundations. Earthquake Engineering \& Structural Dynamics, 11(6):797-808, 1983.

[10] M. Schevenels, G. Lombaert, G. Degrande, and D. Clouteau. The wave propagation in a beam on a random elastic foundation. Probabilistic Engineering Mechanics, 22(2):150-158, 2007.

[11] K.L. Knothe and S.L. Grassie. Modelling of railway track and vehicle/track interaction at high frequencies. Vehicle System Dynamics, 22(3-4):209-262, 1993.

[12] L. ANDERSEN, S.R.K. NIELSEN, and P.H. KIRKEGAARD. Finite element modelling of infinite euler beams on kelvin foundations exposed to moving loads in convected co-ordinates. Journal of Sound and Vibration, 241(4):587-604, 2001.

[13] J. Oscarsson. Simulation of train-track interaction with stochastic track properties. Vehicle System Dynamics, 37(6):449-469, 2002.

[14] Z.G. Li and T.X. Wu. Vehicle/track impact due to passing the transition between a floating slab and ballasted track. In Schulte-Werning, editor, Noise and Vibration Mitigation for Rail Transportation Systems, volume 99 of Notes on Numerical Fluid Mechanics and Multidisciplinary Design, pages 94-100. Springer Berlin Heidelberg, 2008.

[15] J.C.O. Nielsen and J. Oscarsson. Simulation of dynamic train-track interaction with state-dependent track properties. Journal of Sound and Vibration, 275:515-532, 2004.

[16] X. Sheng, M. Li, C.J.C. Jones, and D.J. Thompson. Using the fourier series approach to study interactions between moving wheels and a periodically supported rail. Journal of Sound and Vibration, 303:873-894, 2007.

[17] M.F.M Hussein. Vibration from underground railways. PhD thesis, University of Cambridge,, Cambridge, 2004.

[18] A. Trochanis, R. Chelliah, and J. Bielak. Unified approach for beams on elastic foundations under moving loads. Journal of Geotechnical Engineering, 113(8):879-895, 1987.

[19] M.F.M Hussein and H.E.M. Hunt. Modelling of floating-slab tracks with continuous slabs under oscillating moving loads. Journal of Sound and Vibration, 297:37-54, 2006.

[20] K-J. Bathe. Finite element procedures. Prentice Hall, Inc., 1996.

[21] J.R. Rieker, Y-H. Lin, and M.W. Trethewey. Discretization considerations in moving load finite element beam models. Finite Elements in Analysis and Design, 21(3):129-144, 1996.

[22] K-J Bathe and M.M.I. Baig. On a composite implicit time integration procedure for nonlinear dynamics. Computers \& Structures, 83:2513-2524, 2005. 